\title{
A semi-implicit discrete-continuum coupling method for porous media based on the effective stress principle at finite strain
}

Kun Wang · WaiChing Sun

5 Received: February 13, 2016/ Accepted: date

Assistant Professor, Department of Civil Engineering and Engineering Mechanics, Columbia University , 614 SW Mudd, Mail Code: 4709, New York, NY 10027 Tel.: 212-854-3143, Fax: 212-854-6267, E-mail: wsun@columbia.edu 
of numerous finite element models that employ the effective stress principle [Prevost, 1982, Simon et al., 1986, Borja and Alarcón, 1995, Armero, 1999, Sun et al., 2013b, Sun, 2015]. Nevertheless, modeling the complex path-dependent responses for geomaterials remains a big challenge [Dafalias and Manzari, 2004]. This difficulty is partly due to the need to incorporate a large amount of internal variables and material parameters, which makes the calibration more difficult. Another difficulty is due to the weak underpinning of the phenomenological approach to replicate anisotropy caused by changes of micro-structures and fabric [Li and Dafalias, 2015].

A conceptually simple but computational expensive remedy to resolve this issue is to explicitly model the microscopic fluid-solid interaction. In fact, this approach has been widely used to study sedimentation problems. Previous work, such as Han et al. [2007], Mansouri et al. [2009], Goniva et al. [2010], Han and Cundall [2013], Robinson et al. [2014], Berger et al. [2015], has obtained various degree of successes in simulating fluidized granular beds by establishing information exchange mechanism among discrete element model and fluid solvers. For a subset of two-phase problems in which the length scale of interest is larger than the grain diameter and the fluid flow is laminar, the pore-scale interstitial fluid motion is often not resolved but instead modeled via a locally averaged Navier-Stokes equations (LANS) that couples with DEM via a parametric drag force [Curtis and Van Wachem, 2004, Robinson et al., 2014]. By assuming that a weak separation of scale is valid, this meso-scale approach essentially couples the large scale Navier-Stokes fluid motion with grain-scale DEM model that captures the granular flow nature via interface force. While this method is found to be very efficient for mixing problem, coupling the macroscopic flow at meso-scale via force-based interaction is not without limitations. First, the simulated hydro-mechanical coupling effect is highly sensitive to the fluid drag force model chosen to replicate the particle-fluid interaction. This can lead to complications for calibration and material identification, as the expressions of these fluid drag forces are often empirically correlated by the local porosity, Reynolds number and other factors such as the diameter of the particles, [Zhu et al., 2007]. Furthermore, the meso-scale fluid-particle simulations still require significant computational resources when a large number of particles are involved.

The purpose of this study is to propose a new multiscale hydro-mechanical model that (1) provides the physical underpinning from discrete element simulations, (2) resolves the problems associated with the phenomenological nature of drag force, and (3) improves the efficiency of large-scale problems. Our target is a sub-class of problems in which the solid skeleton is composed of particulate assemblies in solid state (rather than granular flow) and the porous space is fully saturated with a single type of pore fluid in laminar regime. As in the previous work for particle-fluid system [Curtis and Van Wachem, 2004, Robinson et al., 2014], we also adopt the assumption that a weak separation of scale exists between the motion of solid particles and that of the pore fluid. Our major departure is the way we employ this weak separation of the pore fluid to establish hydro-mechanical coupling across length scales. Instead of using the phenomenological drag force model to establish coupling, we use the effective stress principle to partition the macroscopic total stress as the sum of effective stress, which comes from microscopic DEM simulation, and the fluid contribution, which comes from the Biot's coefficient inferred from DEM assemblies and the pore pressure updated from a total Lagrangian poromechanics finite element solver.

The coupled transient problem requires a time integration scheme to advance numerical solution from known solid displacement $\boldsymbol{u}_{n}$ and pore-fluid pressure $p_{n}^{f}$ at time $t_{n}$ to unknowns $\boldsymbol{u}_{n+1}$ and $p_{n+1}^{f}$ at the next time step $t_{n+1}=t_{n}+\Delta t$. Explicit integration scheme has been employed in multiscale dynamic analysis of soils [Onate and Rojek, 2004]. This method is simple in the sense that it advances solutions without solving system of equations. However, it often requires small time steps in order to achieve numerical stability, and when coupling with DEM solver, the condition is even more stringent. Another approach, the implicit scheme, has the possibility of attaining unconditional stability, but the linearization of variational equations, equation solving and iterations require much computational cost per time step. To make a trade-off, Hughes et al. [1979] and Prevost [1983] suggest the usage of an implicit-explicit predictor/multicorrector scheme in nonlinear hydro-mechanical transient problem. Our main contribution in this study is the extension of this method to multiscale coupling problems. We suggest a distinct treatment of the elastic and plastic component of material stiffness homogenized from DEM microstructures. Accordingly, an information exchange scheme is established between the FEM and DEM solvers.

The rest of this paper is organized as follows. In section 2, we first describe the homogenization theory for saturated porous media serving as the framework for micro-macro transitions. Then, the discretecontinuum coupling model in the finite deformation range is presented in Section 3. The details of the 
multiscale semi-implicit method are provided in Section 4, with an emphasis placed on how the material properties homogenized from DEM are employed in the semi-implicit FEM-mixed-DEM solution scheme. Selected problems in geomechanics are simulated via the proposed method to study its performance and their results are presented in Section 5. Finally, concluding remarks are given in Section 6.

As for notations and symbols, bold-faced letters denote tensors; the symbol ' $\because$ ' denotes a single contraction of adjacent indices of two tensors (e.g. $\boldsymbol{a} \cdot \boldsymbol{b}=a_{i} b_{i}$ or $\boldsymbol{c} \cdot \boldsymbol{d}=c_{i j} d_{j k}$ ); the symbol ' $:$ ' denotes a double contraction of adjacent indices of tensor of rank two or higher ( e.g. $C: \epsilon^{e}=C_{i j k l} \epsilon_{k l}^{e}$ ); the symbol ' $\otimes$ ' denotes a juxtaposition of two vectors (e.g. $\boldsymbol{a} \otimes \boldsymbol{b}=a_{i} b_{j}$ ) or two symmetric second order tensors (e.g. $\left.(\boldsymbol{\alpha} \otimes \boldsymbol{\beta})=\alpha_{i j} \beta_{k l}\right)$. As for sign conventions, we consider the direction of the tensile stress and dilative pressure as positive. We impose a superscript $(\cdot)^{\mathrm{DEM}}$ on a variable to emphasize that such variable is inferred from DEM.

\section{Homogenization theory for porous media}

In this section, we describe the homogenization theory we adopt to establish the DEM-mixed-FEM coupling model for fully saturated porous media. Previous work for dry granular materials, such as Miehe and Dettmar [2004], Miehe et al. [2010], Nitka et al. [2011], Guo and Zhao [2014], has demonstrated that a hierarchical discrete-continuum coupling model can be established by using grain-scale simulations to provide Gauss point stress update for finite element simulations in a fully implicit scheme. Nevertheless, the extension of this idea for partially or fully saturated porous media has not been explored, to the best knowledge of the authors.

In this work, we hypothesize that the pore-fluid flow inside the pores is in the laminar regime and is dominated by viscous forces such that Darcy's law is valid at the representative elementary volume level [Sun et al., 2011b,a, 2013a]. Provided that this assumption is valid, we define the pore pressure field only at the macroscopic level and neglect local fluctuation of the pore pressure at the pore- and grain-scale.

On the other hand, we abandon the usage of macroscopic constitutive law to replicate the constitutive responses of the solid constituent. Instead, we apply the effective stress principle [Terzaghi et al., 1943, Gray et al., 2009, 2013] and thus allow the change of the macroscopic effective stress as a direct consequence of the compression, deformation and shear resistance of the solid constituent inferred from grain-scale simulations. As a result, the effective stress can be obtained from homogenizing the forces and branch vectors of the force network formed by the solid particles or aggregates, while the total stress becomes a partition of the homogenized effective stress from the microscopic granular assemblies, and the pore pressure from the macroscopic mixture continuum.

\subsection{Dual-scale effective stress principle}

In this study, we make assumptions that (1) a separation of scale exists and that (2) a representative volume element (RVE) can be clearly defined. Strictly speaking, the assumption (2) is true if the unit cell has a periodic microstructure or when the volume is sufficiently large such that it possesses statistically homogeneous and ergodic properties [Gitman et al., 2007].

With the aforementioned assumptions in mind, we consider a homogenized macroscopic solid skeleton continuum $\mathcal{B}^{s} \subset \mathbb{R}^{3}$ whose displacement field is $C^{0}$ continuous. Each position of the macroscopic solid body in the reference configuration, i.e., $\boldsymbol{X}=\boldsymbol{X}^{s} \in \mathcal{B}_{0}^{s}$, is associated with a micro-structure of the RVE size. Let us denote the trajectories of the macroscopic solid skeleton and the fluid constituent in the saturated two-phase porous medium from the reference configuration to the current solid configuration as,

$$
x=\varphi^{S}(X, t) ; x=\varphi^{f}\left(X^{f}, t\right)
$$

Unless the porous medium is locally undrained, the solid and fluid constituents are not bundled to move along the same trajectory, i.e., $\varphi^{s}(\cdot, t) \neq \varphi^{f}(\cdot, t)$. If we choose to follow the macroscopic solid skeleton trajectory to formulate the macroscopic balance principles, then the control volumes are attached to solid skeleton only, and the pore fluid motion is described by relative movement between the fluid constituent 
and the solid matrix, as shown in Fig. 1. The deformation gradient of the macroscopic solid constituent $F$ can therefore be written as,

$$
\boldsymbol{F}=\frac{\partial \varphi\left(X^{s}, t\right)}{\partial X^{s}}=\frac{\partial \varphi(X, t)}{\partial X}=\frac{\partial x}{\partial X}
$$

in which we omit the superscript $s$ when quantities are referred to solid phase. Now, following [Miehe

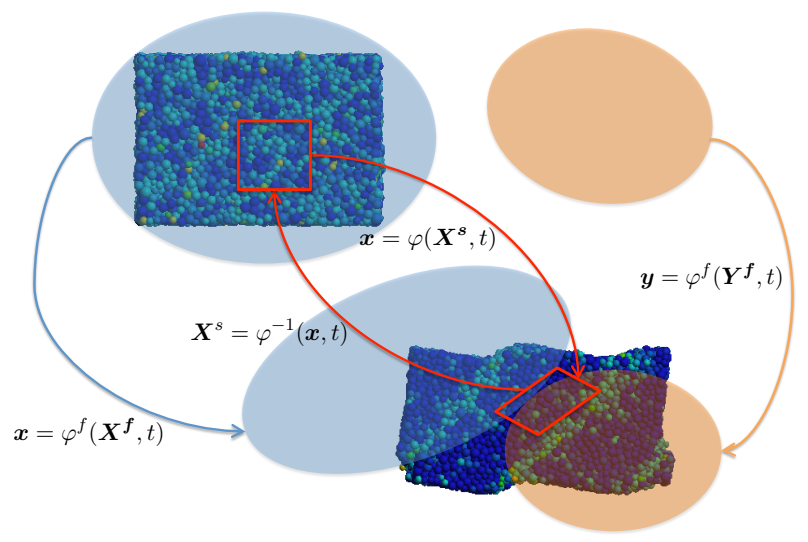

Fig. 1: Trajectories of the solid and fluid constituents $\varphi^{\varsigma}=\varphi$ and $\varphi^{f}$. The motion $\varphi$ conserves all the mass of the solid constituent, while the fluid may enter or leave the body of the solid constituent. Figure reproduced from [Sun et al., 2013b]

and Dettmar, 2004], we associate each point in the current configuration $x$ with an aggregate of $N$ particles inside the representative volume $\mathcal{V}$. Furthermore, we introduce a local coordinate system for the RVE in which the position vector $y \in \mathbb{R}^{3}$ becomes $\mathbf{0}$ at the geometric centroid of the RVE. The locations of the centroids of the $N$ particles expressed using the local coordinate system read, i.e.,

$$
y_{p} \in \mathcal{V}, p=1,2, \ldots N .
$$

where $y_{p}$ is the local position vector of the center of the $p$-th particle in the microstructure and $x+y_{p}$ is the same position expressed in the macroscopic current coordinate system. Particles inside the RVE may make contacts to each other. The local position vector of each contact between each particle-pair $\boldsymbol{y}_{c}$ can be written as,

$$
y_{c} \in \mathcal{V}, c=1,2, \ldots N_{c} .
$$

Both the positions of the particles $y_{p}$ and that of the contacts $y_{c}$ are governed by contact law and the equilibrium equations. Previous works, such as Curtis and Van Wachem [2004], El Shamy and Zeghal [2005], Han and Cundall [2011], Galindo-Torres et al. [2013], Robinson et al. [2014], Cui et al. [2014], have found success in explicitly modeling the pore-scale grain-fluid interaction. Nevertheless, such grain-fluid interaction simulations do impose a very high computational demand due to the fact that the fluid flow typically requires at least an order more of degree of freedoms to resolve the flow in the void space among particles. However, for seepage flow that is within the laminar regime where Darcy's law applies, the new insight obtained from the costly simulations will be limited. As a result, this discrete-continuum coupling model does not explicitly model the pore-scale solid-fluid interaction. Instead, we rely on the hypothesis that effective stress principle is valid for the specific boundary value problems we considered. In particular, we make the following assumptions:

- The void space is always fully saturated with one type of fluid and there is no capillary effect that leads to apparent cohesion of the solid skeleton. 
- The flow in the void space remains Darcian at the macroscopic level.

- All particles in the granular assemblies are in contact with the neighboring particles.

- Fluidization, suffusion and erosion do not occur.

- Grain crushing does not occur.

- There is no mass exchange between the fluid and solid constituents.

As a result, we may express the total macroscopic Cauchy stress as a function of homogenized Cauchy effective stress inferred from DEM and the macroscopic pore pressure obtained from the mixed finite element, i.e.

$$
\boldsymbol{\sigma}(\boldsymbol{x}, t)=<\boldsymbol{\sigma}^{\prime}(\boldsymbol{x}, t)>_{\mathrm{RVE}}-B(\boldsymbol{x}, t) p^{f}(\boldsymbol{x}, \boldsymbol{t}) \boldsymbol{I}
$$

where

$$
<\boldsymbol{\sigma}^{\prime}(\boldsymbol{x}, t)>_{\mathrm{RVE}}=\frac{1}{2 \mathrm{~V}_{\mathrm{RVE}}} \sum_{c}^{N_{c}}\left(\boldsymbol{f}^{\mathcal{c}} \otimes \boldsymbol{l}^{c}+\boldsymbol{l}^{c} \otimes \boldsymbol{f}^{c}\right)
$$

$f^{c}$ is the contact force and $l^{c}$ is the branch vector, the vector that connects the centroids of two grains forming the contact [Christoffersen et al., 1981, Bagi, 1996, Sun et al., 2013a], at the grain contact $\boldsymbol{x}+\boldsymbol{y}_{c} \in$ $\mathbb{R}^{3}$. $V_{\text {RVE }}$ is the volume of the RVE and $N_{c}$ is the total number of particles in the RVE. Meanwhile, the Biot's coefficient $B$ reads,

$$
B(x, t)=1-\frac{K_{T}^{\mathrm{DEM}}(\boldsymbol{x}, t)}{K_{s}}
$$

with $K_{T}^{\mathrm{DEM}}(x, t)$ and $K_{s}$ being the effective tangential bulk modulus of the solid matrix inferred from DEM, and the bulk modulus of the solid grain respectively [Nur and Byerlee, 1971, Simon et al., 1986]. Notice that, in the geotechnical engineering and geomechanics literature, such as $\mathrm{Ng}$ [2006], Kuhn et al. [2014], it is common to impose incompressible volumetric constraint on dry DEM assembly to simulate undrained condition at meso-scale. This treatment can be considered as a special case of (7) when the bulk modulus of the solid grain is significantly higher than that of the skeleton such that the Biot's coefficient is approximately equal to one.

\subsection{Micro-macro-transition for solid skeleton}

In this study, we consider the class of two-phase porous media of which the solid skeleton is composed of particles. These particles can be cohesion-less or cohesive, but the assemblies they formed are assumed to be of particulate nature and hence suitable for DEM simulations. [Cundall and Strack, 1979].

In our implementation, the DEM simulations are conducted via YADE (Yet Another Dynamic Engine [Šmilauer et al., 2010]), an open source code base for discontinua. These grain-scale DEM simulations are used as a replacement to the macroscopic constitutive laws that relate strain measure with effective stress measure for each RVE associated with a Gauss point in the macroscopic mixed finite element. In particular, a velocity gradient is prescribed to move the frame of the unit cell and the DEM will seek for the static equilibrium state via dynamics relaxation method. After static equilibrium is achieved, the internal forces and branch vectors are used to compute the homogenized effective Cauchy stress via the micro-macro transition theory [Miehe and Dettmar, 2004, Miehe et al., 2010, Wellmann et al., 2008]. For completeness, we provide a brief overview of DEM, the procedure for generation of RVEs and the study on the size of RVEs in Appendix A,B and C.

The Hill-Mandel micro-heterogeneity condition demands that the power at the microscopic scale must be equal to the the rate of work done measured by the macroscopic effective stress and strain rate measures. For the solid constituent of the two-phase porous media, this condition can be expressed in terms of any power-conjugate effective stress and strain rate pair, such as $\left(\boldsymbol{P}^{\prime}, \dot{\boldsymbol{F}}\right)$ and $\left(\boldsymbol{S}^{\prime}, \dot{\boldsymbol{E}}\right)$ and $\left(\boldsymbol{\sigma}^{\prime}, \boldsymbol{D}\right)$ [Borja and Alarcón, 1995, Armero, 1999]. For instance, the condition can be written in terms of the effective stress and rate of deformation of the solid skeleton, i.e.,

$$
<\sigma^{\prime}>_{\mathrm{RVE}}:<\boldsymbol{D}>_{\mathrm{RVE}}=<\boldsymbol{\sigma}^{\prime}: \boldsymbol{D}>_{\mathrm{RVE}}
$$

where $\boldsymbol{D}$ is the rate of deformation, i.e., the symmetric part of the velocity gradient tensor,

$$
<\boldsymbol{D}>_{\mathrm{RVE}}=\frac{1}{2}\left(<\boldsymbol{L}>_{\mathrm{RVE}}+<\boldsymbol{L}^{\mathrm{T}}>_{\mathrm{RVE}}\right) ; \boldsymbol{L}=\nabla^{x} v
$$


and $\left\langle\sigma^{\prime}\right\rangle_{\text {RVE }}$ is defined previously in (6). Previous studies, such as, Miehe and Dettmar [2004], Wellmann et al. [2008], Miehe et al. [2010], Fish [2013], have established that the linear deformation, periodic, and uniform traction are three boundary conditions that satisfy the Hill-Mandel micro-heterogeneity condition. In our implementation, we apply the periodic boundary condition to obtain the effective stress measure, because the periodic boundary condition may yield responses that are softer than those obtained from the linear deformation $\mathrm{BC}$ but stiffer than those obtained from the uniform traction $\mathrm{BC}$. In particular, the periodic boundary condition enforces two constraints: (1) the periodicity of the deformation, i.e.,

$$
\left[\left[\boldsymbol{y}_{b}\right]\right]=<\boldsymbol{F}>_{\mathrm{RVE}}\left[\left[\boldsymbol{Y}_{b}\right]\right] \text { and }\left[\left[\boldsymbol{R}_{b}\right]\right]=\mathbf{0}
$$

where [[-]] denotes the jump across boundaries, $\boldsymbol{y}_{b}$ and $\boldsymbol{Y}_{b}$ represent the position vectors of the particles at the boundary of the reference and current configurations, $R_{b} \in S O(3)$ represents the rotation tensor of particles at the boundary, and (2) the anti-periodicity of the force $f_{b}$ and moment on the boundary of the RVE, i.e.,

$$
\left[\left[f_{b}\right]\right]=\mathbf{0} \text { and }\left[\left[\left(y_{c}-y_{b}\right) \times f_{b}\right]\right]=\mathbf{0}
$$

In YADE, the DEM code we employed for grain-scale simulations, the deformation of an RVE is driven by a periodic cell box in which the macroscopic velocity gradient of the unit cell $<L>_{\mathrm{RVE}}$ can both be measured and prescribed.

\section{Multiscale DEM-mixed-FEM hydro-mechanical model}

The differential equations governing the isothermal saturated porous media in large deformation are derived based on the mixture theory, in which solid matrix and pore fluid are treated together as a multiphase continuum [Prevost, 1982, Borja and Alarcón, 1995, Armero, 1999, Coussy, 2004, Sun et al., 2013b, Martinez et al., 2013]. The solid and fluid constituents may simultaneously occupy fractions of the volume of the same material point. The physical quantities of the mixture, such as density and total stress, are spatially homogenized from its components. For example, the averaged density of the fluid saturated soil mixture is defined as:

$$
\rho=\rho^{s}+\rho^{f}=(1-\phi) \rho_{s}+\phi \rho_{f}
$$

where $\rho^{\alpha}$ is the partial mass density of the $\alpha$ constituent and $\rho_{\alpha}$ is the intrinsic mass density of the $\alpha$ constituent, with $\phi$ being the porosity.

\subsection{Balance of linear momentum}

For the balance of linear momentum law in finite strain, we adopt the total Lagrangian formulation and choose the total second Piola-Kirchhoff stress (PK2) $S$ as the stress measure. The inertial effect is neglected. The equation takes the form:

$$
\nabla^{X} \cdot(\boldsymbol{F S})+J\left(\rho^{s}+\rho^{f}\right) \boldsymbol{g}=\mathbf{0}
$$

where the Jacobian $J=\operatorname{det}(\boldsymbol{F})$. The principle of effective stress postulates that the total Cauchy stress $\sigma$ can be decomposed into an effective stress due to the solid skeleton deformation and an isotropic pore pressure $\left(p^{f}\right)$ stress. The effective stress principle in terms of PK2 writes:

$$
\boldsymbol{S}=\boldsymbol{S}^{\mathrm{DEM}}-J \boldsymbol{F}^{-1} B^{\mathrm{DEM}} p^{f} \boldsymbol{I} \boldsymbol{F}^{-T}
$$

where

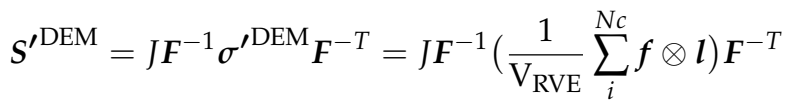

Thus the balance of linear momentum becomes:

$$
\nabla^{\boldsymbol{X}} \cdot\left(\boldsymbol{F} \boldsymbol{S}^{\prime \mathrm{DEM}}-J B^{\mathrm{DEM}} p^{f} \boldsymbol{F}^{-T}\right)+J\left(\rho^{s}+\rho^{f}\right) \boldsymbol{g}=\mathbf{0}
$$


3.2 Balance of fluid mass

The simplified $\mathrm{u}-\mathrm{p}$ formulation in finite strain requires another equation illustrating the balance of mass for pore fluid constituent:

$$
\frac{D \rho^{f}}{D t}=-\nabla^{X} \cdot\left(J \boldsymbol{F}^{-\mathbf{1}}\left[\phi^{\mathrm{DEM}} \rho_{f}\left(\boldsymbol{v}^{f}-\boldsymbol{v}\right)\right]\right)
$$

where $\frac{D[.]}{D t}=[$.$] is the material time derivative with respect to the velocity of solid skeleton v$.

We make isothermal and barotropic assumptions and suppose that $p^{f}<<K_{s}$ and that $\frac{D B^{D E M}}{D t} \sim 0$. After simplifications [Sun et al., 2013b], the balance of mass becomes:

$$
\frac{B^{\mathrm{DEM}}}{J} \frac{D J}{D t}+\frac{1}{M^{\mathrm{DEM}}} \frac{D p^{f}}{D t}+\nabla^{X} \cdot\left(\frac{1}{\rho_{f}}\left(J \boldsymbol{F}^{-\mathbf{1}}\left[\phi^{\mathrm{DEM}} \rho_{f}\left(\boldsymbol{v}^{f}-\boldsymbol{v}\right)\right]\right)\right)=0
$$

where

$$
M^{\mathrm{DEM}}=\frac{K_{s} K_{f}}{K_{f}\left(B^{\mathrm{DEM}}-\phi^{\mathrm{DEM}}\right)+K_{s} \phi^{\mathrm{DEM}}}
$$

is the Biot's modulus [Nur and Byerlee, 1971], with $K_{f}$ being the bulk modulus of pore fluid.

In this paper, Darcy's constitutive law relating the relative flow and the pore pressure is employed, neglecting the inertial effect:

$$
\boldsymbol{Q}=\boldsymbol{K}^{\mathrm{DEM}} \cdot\left(-\nabla^{\mathrm{X}} p^{f}+\rho_{f} \boldsymbol{F}^{\mathrm{T}} \cdot \boldsymbol{g}\right)
$$

where the pull-back permeability tensor $\boldsymbol{K}^{\mathrm{DEM}}$ is defined as

$$
\boldsymbol{K}^{\mathrm{DEM}}=J \boldsymbol{F}^{-1} \cdot \boldsymbol{k}^{\mathrm{DEM}} \cdot \boldsymbol{F}^{-\mathrm{T}}
$$

Assume that the effective permeability tensor $\boldsymbol{k}^{\mathrm{DEM}}$ is isotropic, i.e.,

$$
\boldsymbol{k}^{\mathrm{DEM}}=k^{\mathrm{DEM}} \boldsymbol{I}
$$

where $k^{\mathrm{DEM}}$ is the scalar effective permeability in unit of $\frac{m^{2}}{P a \cdot s}$. It is updated from porosity of DEM RVEs according to the Kozeny-Carmen equation.

\subsection{Weak form}

To construct the macroscopic hydro-mechanical boundary-value problem, consider a reference domain $\mathcal{B}$ with its boundary $\partial \mathcal{B}$ composed of Dirichlet boundaries (solid displacement $\partial \mathcal{B}_{u}$, pore pressure $\partial \mathcal{B}_{p}$ ) and Von Neumann boundaries (solid traction $\partial \mathcal{B}_{t}$, fluid flux $\partial \mathcal{B}_{q}$ ) satisfying

$$
\left\{\begin{aligned}
\partial \mathcal{B} & =\overline{\partial \mathcal{B}_{u} \cup \partial \mathcal{B}_{t}}=\overline{\partial \mathcal{B}_{p} \cup \partial \mathcal{B}_{q}} \\
\varnothing & =\partial \mathcal{B}_{u} \cap \partial \mathcal{B}_{t}=\partial \mathcal{B}_{p} \cap \partial \mathcal{B}_{q}
\end{aligned}\right.
$$

The prescribed boundary conditions are

$$
\left\{\begin{aligned}
\boldsymbol{u} & =\bar{u} \text { on } \partial \mathcal{B}_{u} \\
\boldsymbol{P} \cdot \boldsymbol{N}=(\boldsymbol{F} \cdot \boldsymbol{S}) \cdot \boldsymbol{N} & =\overline{\boldsymbol{t}} \text { on } \partial \mathcal{B}_{t} \\
p^{f} & =\bar{p} \text { on } \partial \mathcal{B}_{p} \\
-\boldsymbol{N} \cdot Q & =\bar{Q} \text { on } \partial \mathcal{B}_{Q}
\end{aligned}\right.
$$

where $N$ is outward unit normal on undeformed surface $\partial \mathcal{B}$.

For model closure, the initial conditions are imposed as

$$
p^{f}=p_{0}^{f}, \boldsymbol{u}=\boldsymbol{u}_{0} \text { at } t=t_{0}
$$


Following the standard procedures of the variational formulation, we obtain finally the weak form of the balance of linear momentum and mass

$$
\begin{aligned}
G: & V_{\boldsymbol{u}} \times V_{p} \times V_{\eta} \rightarrow \mathbb{R} \\
G\left(\boldsymbol{u}, p^{f}, \boldsymbol{\eta}\right)= & \int_{\mathcal{B}} \nabla^{\boldsymbol{X}} \boldsymbol{\eta}:\left(\boldsymbol{F} \cdot \boldsymbol{S}^{\prime \mathrm{DEM}}-J B p^{f} \boldsymbol{F}^{-\mathrm{T}}\right) \mathrm{dV}-\int_{\mathcal{B}} J\left(\rho^{f}+\rho^{s}\right) \boldsymbol{\eta} \cdot \boldsymbol{g d V} \\
& -\int_{\partial \mathcal{B}_{t}} \boldsymbol{\eta} \cdot \overline{\boldsymbol{t}} \mathrm{d} \Gamma=0
\end{aligned}
$$

$$
\begin{aligned}
H: & V_{\boldsymbol{u}} \times V_{p} \times V_{\psi} \rightarrow \mathbb{R} \\
H\left(\boldsymbol{u}, p^{f}, \psi\right)= & \int_{\mathcal{B}} \psi \frac{B^{\mathrm{DEM}}}{J} \dot{j} \mathrm{dV}+\int_{\mathcal{B}} \psi \frac{1}{M^{\mathrm{DEM}}} \dot{p}^{f} \mathrm{dV} \\
& -\int_{\mathcal{B}} \nabla^{\boldsymbol{X}} \psi \cdot\left[\boldsymbol{K}^{\mathrm{DEM}} \cdot\left(-\nabla^{\boldsymbol{X}} p^{f}+\rho_{f} \boldsymbol{F}^{\mathrm{T}} \cdot \boldsymbol{g}\right)\right] \mathrm{dV} \\
& -\int_{\partial B_{\mathcal{Q}}} \psi \overline{\mathbf{Q}} \mathrm{d} \Gamma=0
\end{aligned}
$$

The first integral of $H\left(\boldsymbol{u}, p^{f}, \psi\right)$ can be related to the solid velocity field $\dot{\boldsymbol{u}}$ using the equations $\dot{J}=J \nabla^{x} \cdot \dot{\boldsymbol{u}}$ and $\nabla^{x} \cdot \dot{u}=\nabla^{X} \dot{u}: F^{-T}$ [Borja and Alarcón, 1995]:

$$
\int_{\mathcal{B}} \psi \frac{B^{\mathrm{DEM}}}{J} \dot{J} \mathrm{dV}=\int_{\mathcal{B}} \psi B^{\mathrm{DEM}} \nabla^{x} \cdot \dot{\boldsymbol{u}} \mathrm{dV}=\int_{\mathcal{B}} \psi B^{\mathrm{DEM}} \boldsymbol{F}^{-\mathrm{T}}: \nabla^{\mathrm{X}} \dot{\boldsymbol{u}} \mathrm{dV}
$$

The displacement and pore pressure trial spaces for the weak form are defined as

$$
V_{\boldsymbol{u}}=\left\{\boldsymbol{u}: \mathcal{B} \rightarrow \mathbb{R}^{3}\left|\boldsymbol{u} \in\left[H^{1}(\mathcal{B})\right]^{3}, \boldsymbol{u}\right|_{\partial B_{u}}=\overline{\boldsymbol{u}}\right\}
$$

$$
V_{p}=\left\{p^{f}: \mathcal{B} \rightarrow \mathbb{R}\left|p^{f} \in H^{1}(\mathcal{B}), p^{f}\right|_{\partial B_{p}}=\bar{p}\right\}
$$

and the corresponding admissible spaces of variations are defined as

$$
V_{\boldsymbol{\eta}}=\left\{\boldsymbol{\eta}: \mathcal{B} \rightarrow \mathbb{R}^{3}\left|\boldsymbol{\eta} \in\left[H^{1}(\mathcal{B})\right]^{3}, \boldsymbol{\eta}\right|_{\partial B_{u}}=\mathbf{0}\right\}
$$

$$
V_{\psi}=\left\{\psi: \mathcal{B} \rightarrow \mathbb{R}\left|\psi \in H^{1}(\mathcal{B}), \psi\right|_{\partial B_{p}}=0\right\}
$$

$H^{1}$ denotes the Sobolev space of degree one, which is the space of square integrable function whose weak derivative up to order 1 are also square integrable (cf. Hughes [1987], Brenner and Scott [2008]).

\subsection{Finite element spatial discretization}

The spatially discretized equations can be derived following the standard Galerkin procedure. Shape functions $N_{u}(X)$ and $N_{p}(X)$ are used for approximation of solid motion $u, \dot{u}$ and pore pressure $p^{f}, \dot{p}^{f}$, respectively:

$$
\left\{\begin{aligned}
u & =N_{u} \bar{u}, \dot{u}=N_{u} \dot{\bar{u}}, \eta=N_{u} \bar{\eta} \\
p^{f} & =N_{p} \bar{p}^{f}, \dot{p}^{f}=N_{p} \dot{\bar{p}}^{f}, \quad \psi=N_{p} \bar{\psi}
\end{aligned}\right.
$$

with $\bar{u}$ being the nodal solid displacement vector, $\bar{p}^{f}$ being the nodal pore pressure vector, $\dot{\bar{u}}^{\prime} \dot{\bar{p}}^{f}$ being their time derivatives, and $\bar{\eta}, \bar{\psi}$ being their variations.

The adopted eight-node hexahedral element interpolates the displacement and pore pressure field with the same order. As a result, this combination does not inherently satisfy the inf-sup condition [White and Borja, 2008, Sun et al., 2013b, Sun, 2015]. Therefore a stabilization procedure is necessary. In this study, the fluid pressure Laplacian scheme is applied. This scheme consists of adding the following stabilization term to the balance of mass equation (27) :

$$
\int_{\mathcal{B}} \nabla^{X} \psi \alpha_{\text {stab }} \nabla^{X} \dot{p}^{f} \mathrm{dV}
$$


with $\alpha_{s t a b}$ a scale factor depending on element size and material properties of the porous media. For detailed formulations, please refer to [Truty and Zimmermann, 2006, Sun et al., 2013b].

We obtain the finite element equations for balance of linear momentum and balance of mass as:

$$
\left\{\begin{array} { r l } 
{ G ( \boldsymbol { u } , p ^ { f } , \boldsymbol { \eta } ) } & { = 0 } \\
{ H ( \boldsymbol { u } , p ^ { f } , \psi ) } & { = 0 }
\end{array} \Longrightarrow \left\{\begin{array}{rl}
\boldsymbol{F}_{\text {int }}^{s}(\overline{\boldsymbol{u}})-\boldsymbol{K}^{u p} \overline{\boldsymbol{p}}^{f}-G^{\mathbf{1}} & =\boldsymbol{F}_{\text {ext }}^{\mathbf{1}} \\
\boldsymbol{C}_{\mathbf{1}} \dot{\bar{u}}+\left(\boldsymbol{C}_{2}+\boldsymbol{C}_{\text {stab }}\right) \dot{\bar{p}}^{f}+\boldsymbol{K}^{p} \bar{p}^{f}-G^{\mathbf{2}} & =\boldsymbol{F}_{\text {ext }}^{2}
\end{array}\right.\right.
$$

with expressions for each term:

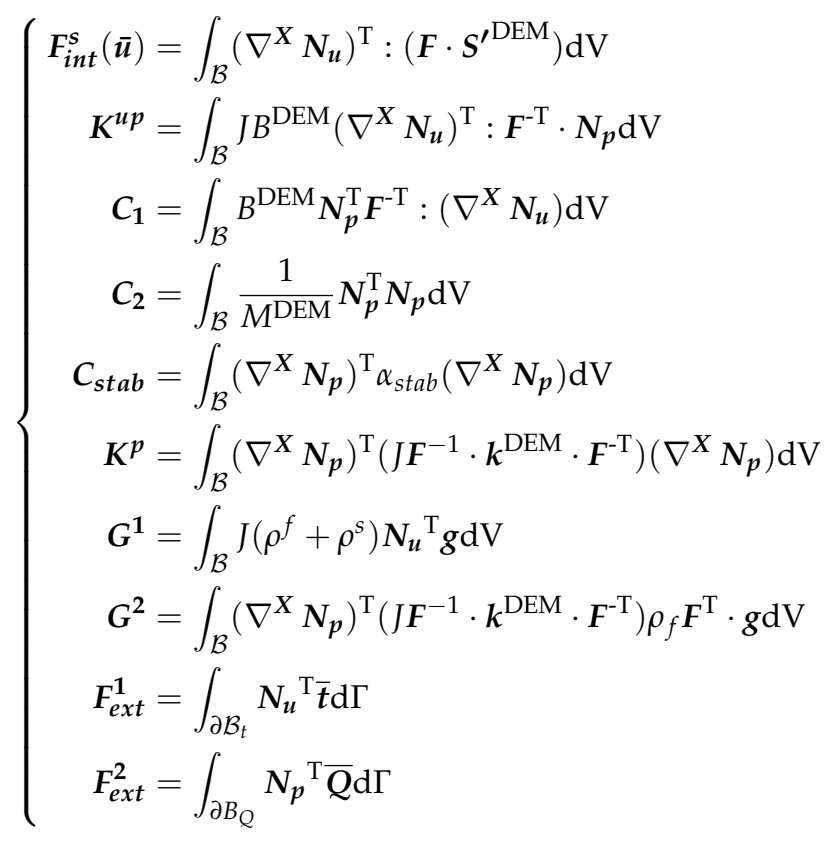

The non-linear equation system (35) can be rewritten in a compact form:

$$
M^{*} \cdot v+F^{i n t}(d)-G(d)=F^{e x t}
$$

$$
\begin{aligned}
& \text { where } M^{*}=\left[\begin{array}{cc}
0 & 0 \\
C_{1}\left(C_{2}+C_{\text {stab }}\right)
\end{array}\right], v=\left\{\begin{array}{c}
\dot{\bar{u}} \\
\dot{\bar{p}}^{f}
\end{array}\right\}, F^{\text {int }}=\left\{\begin{array}{c}
F_{\text {int }}^{s}(\bar{u})-K^{u p} \bar{p}^{f} \\
K^{p} \bar{p}^{f}
\end{array}\right\}, d=\left\{\begin{array}{c}
\bar{u} \\
\bar{p} f
\end{array}\right\}, G=\left\{\begin{array}{l}
G^{1} \\
G^{2}
\end{array}\right\} \text { and } \\
& F^{\text {ext }}=\left\{\begin{array}{l}
F_{\text {ext }}^{1} \\
F_{\text {ext }}^{2}
\end{array}\right\} .
\end{aligned}
$$

\subsection{Consistent linearization}

The semi-implicit solution scheme requires the expression of the tangential stiffness of the implicit contribution. Thus, we perform the consistent linearization of the weak forms (26) and (27) in the reference configuration [Borja et al., 1998, Sanavia et al., 2002]. For the balance of linear momentum equation, the 
consistent linearization reads,

$$
\begin{aligned}
& \delta G\left(\boldsymbol{u}, p^{f}, \boldsymbol{\eta}\right)=\overbrace{\int_{\mathcal{B}}\left(\boldsymbol{F}^{\mathrm{T}} \cdot \nabla^{X} \boldsymbol{\eta}\right):\left(\boldsymbol{C}^{S E}\right)^{\mathrm{DEM}}: \delta \boldsymbol{E d V}}^{\overline{\boldsymbol{\eta}}^{\mathrm{T}} \boldsymbol{K}^{s} \delta \bar{u}}+\overbrace{\int_{\mathcal{B}}{\boldsymbol{S}^{\prime}}^{\mathrm{DEM}}:\left(\nabla^{X} \delta \boldsymbol{u}\right)^{\mathrm{T}} \cdot \nabla^{X} \eta \mathrm{dV}}^{\bar{\eta}^{\mathrm{T}} \boldsymbol{K}_{S^{\prime}}^{g e o} \delta \overline{\boldsymbol{u}}} \\
& -\overbrace{\int_{\mathcal{B}} \nabla^{X} \eta: \delta\left(J B^{\mathrm{DEM}} \boldsymbol{F}^{-\mathrm{T}}\right) p^{f} \mathrm{dV}}^{\bar{\eta}^{\mathrm{T}} \boldsymbol{K}_{p f}^{g e o} \delta \bar{u}}-\overbrace{\int_{\mathcal{B}} J B^{\mathrm{DEM}} \nabla^{X} \eta: \boldsymbol{F}^{-\mathrm{T}} \delta p^{f} \mathrm{dV}}^{\bar{\eta}^{\mathrm{T}} \boldsymbol{K}^{u p} \delta \bar{p}^{f}} \\
& -\overbrace{\int_{\mathcal{B}} \rho_{f} \nabla^{X} \cdot\left(J \boldsymbol{F}^{-1} \cdot \delta \boldsymbol{u}\right) \boldsymbol{\eta} \cdot \boldsymbol{g d V}}^{\bar{\eta}^{\mathrm{T}} \delta G^{1}}-\overbrace{\int_{\partial \mathcal{B}_{t}}^{\eta} \boldsymbol{\eta} \cdot \delta \bar{t} \mathrm{~d} \Gamma}^{\bar{\eta}^{\mathrm{T}} \delta \boldsymbol{F}_{\text {ext }}^{1}}=0
\end{aligned}
$$

where $C_{I J K L}^{S E}=\frac{\partial S_{I J}^{\prime}}{\partial E_{K L}}$ is the material tangential stiffness. $\delta E$ is the variation of the Green-Lagrange strain tensor and $\delta \boldsymbol{E}=\frac{1}{2}\left[\left(\nabla^{\boldsymbol{X}} \delta \boldsymbol{u}\right)^{\mathrm{T}} \boldsymbol{F}+\boldsymbol{F}^{\mathrm{T}}\left(\nabla^{\boldsymbol{X}} \delta \boldsymbol{u}\right)\right] . \boldsymbol{K}_{\boldsymbol{S}^{\prime}}^{\text {geo }}$ and $\boldsymbol{K}_{p^{f}}^{\text {geo }}$ are the initial stress and initial pore pressure contributions to the geometrical stiffness. For the balance of mass equation, the corresponding linearization term reads,

$$
\begin{aligned}
& \delta H\left(\boldsymbol{u}, p^{f}, \psi\right)=\overbrace{\int_{\mathcal{B}} \psi \delta\left(B^{\mathrm{DEM}} \boldsymbol{F}^{-\mathrm{T}}\right): \nabla^{X} \dot{\boldsymbol{u}} \mathrm{dV}}^{\bar{\psi}^{\mathrm{T}} \delta C_{\dot{1}} \dot{\bar{u}}}+\overbrace{\int_{\mathcal{B}} \psi B^{\mathrm{DEM}} \boldsymbol{F}^{-\mathrm{T}}: \nabla^{X} \delta \dot{\boldsymbol{u}} \mathrm{dV}}^{\bar{\psi}^{\mathrm{T}} C_{1} \delta \dot{\bar{u}}} \\
& +\overbrace{\int_{\mathcal{B}} \psi \delta\left(\frac{1}{M^{\mathrm{DEM}}}\right) \dot{p}^{f} \mathrm{dV}}^{\bar{\psi}^{\mathrm{T}} \delta C_{2} \dot{\bar{p}}^{f}}+\overbrace{\int_{\mathcal{B}} \psi \frac{1}{M^{\mathrm{DEM}}} \delta \dot{p}^{f} \mathrm{dV}}^{\bar{\psi}^{\mathrm{T}} C_{2} \delta \dot{\bar{p}}^{f}}+\overbrace{\int_{\mathcal{B}} \nabla^{X} \psi \alpha_{s t a b} \nabla^{X} \delta \dot{p}^{f} \mathrm{dV}}^{\bar{\psi}^{\mathrm{T}} C_{s t a b} \delta \dot{\bar{p}}^{f}} \\
& +\overbrace{\int_{\mathcal{B}} \nabla^{X} \psi \cdot \boldsymbol{K}^{\mathrm{DEM}} \cdot \nabla^{X} \delta p^{f} \mathrm{dV}}^{\bar{\psi}^{\mathrm{T}} \boldsymbol{K}^{p} \delta \bar{p}^{-f}}+\overbrace{\int_{\mathcal{B}} \nabla^{X} \psi \cdot \delta \boldsymbol{K}^{\mathrm{DEM}} \cdot \nabla^{X} p^{f} \mathrm{dV}}^{\bar{\psi}^{\mathrm{T}} \boldsymbol{K}_{1}^{p} \delta \bar{u}} \\
& -\overbrace{\int_{\mathcal{B}} \nabla^{X} \psi \cdot \delta\left(\boldsymbol{K}^{\mathrm{DEM}} \cdot \rho_{f} \boldsymbol{F}^{\mathrm{T}}\right) \cdot g \mathrm{dV}}^{\bar{\psi}^{\mathrm{T}} \delta G^{2}}-\overbrace{\int_{\partial B_{Q}} \psi \delta \overline{\boldsymbol{Q}} \mathrm{d} \Gamma}^{\bar{\psi}^{\mathrm{T}} \delta F_{\text {ext }}^{2}}=0
\end{aligned}
$$

where $K_{1}^{p}$ is the geometrical term related to the permeability $k$.

The proposed semi-implicit scheme splits $G\left(\boldsymbol{u}, p^{f}, \boldsymbol{\eta}\right)$ and $H\left(\boldsymbol{u}, p^{f}, \psi\right)$ into implicitly treated parts and explicitly treated parts, thus only a subset of the linearization terms in (38) and (39) will be used. The implicit-explicit split will be explained in the next section.

\section{Semi-implicit multiscale time integrator}

While both implicit and explicit time integrators have been used in DEM-FEM coupling models for dry granular materials [Miehe and Dettmar, 2004, Guo and Zhao, 2014, Liu et al., 2015], the extension of these algorithms to multiphysics hydro-mechanical problem is not straightforward. The key difference is that the pore-fluid diffusion is transient and hence the initial boundary value problem is elliptic.

While it is possible to add the inertial terms and update the macroscopic displacement and pore pressure explicitly via a dynamics relaxation procedure, this strategy is impractical due to the small critical time step size of the explicit scheme as pointed out by Prevost [1983]. Another possible approach is to solve the macroscopic problem in a fully implicit, unconditionally stable scheme. The drawback of this approach is that it requires additional CPU time to compute the elasto-plastic tangential stiffness from DEM. Unlike a conventional constitutive model (in which an analytical expression of the tangential stiffness is often available and hence easy to implement), the tangential stiffness inferred from DEM must be obtained numerically via perturbation methods [Guo and Zhao, 2014, Brothers et al., 2014]. For three-dimensional simulations, this means that additional 36 to 81 simulations are required to obtain the tangential stiffness, 
depending on which energy-conjugate stress-strain pair is used in the formulation. This is a sizable burden given the fact that a converged update may require tens of iterations.

To avoid this additional computational cost, we adopt the implicit-explicit predictor/multicorrector scheme originally proposed in Hughes et al. [1979] and Prevost [1983] and apply it to the finite strain DEM-mixed-FEM model. In Prevost [1983], the internal force is split into two components, one treated implicitly and another treated explicitly. We adopt this idea here by treating the elasto-plastic force from DEM explicitly, and the other internal forces implicitly, in a fashion similar to the unconditionally stable Yanenko operator splitting (i.e. $\mathcal{L}=\mathcal{L}_{\exp }+\mathcal{L}_{\text {imp }}$, c.f. Yanenko [1971]).

The implicit time integration based on the generalized trapezoidal rule consists of satisfying the equation (37) at time $t_{n+1}$ :

$$
M_{n+1}^{*} \cdot v_{n+1}+F^{i n t}\left(d_{n+1}\right)-G\left(d_{n+1}\right)=F_{n+1}^{e x t}
$$

with the solution

$$
d_{n+1}=\tilde{d}+\alpha \Delta t v_{n+1}
$$

where

$$
\tilde{d}=d_{n}+(1-\alpha) \Delta t v_{n} .
$$

The notation is as follows: the subscripts $n$ and $n+1$ denote that the variables are evaluated at time $t_{n}$ and $t_{n+1}$, respectively; $\Delta t$ is the time step; $\alpha$ is the integration parameter. The quantity $\tilde{d}$ is referred to as the predicted solution.

Similar to the scheme of Prevost [1983], the semi-implicit predictor-corrector scheme is performed by evaluating a portion of the left hand side forces of (40) explicitly using the predicted solution $\tilde{d}$ and $v_{n}$, and treating the remaining portion implicitly with the solution $d_{n+1}$ and $v_{n+1}$ :

$$
F_{\mathrm{IMP}}\left(v_{n+1}, d_{n+1}\right)+F_{\mathrm{EXP}}\left(v_{n}, \tilde{d}\right)=F_{n+1}^{e x t}
$$

where

$$
\left\{\begin{array}{l}
\boldsymbol{F}_{\mathrm{IMP}}=\left\{\boldsymbol{M}^{*} \cdot \boldsymbol{v}\right\}_{n+1}^{\text {implicit }}+\left\{\boldsymbol{F}^{\text {int }}\left(\boldsymbol{d}_{\boldsymbol{n}+1}\right)\right\}^{\text {implicit }} \\
\boldsymbol{F}_{\mathrm{EXP}}=\left\{\boldsymbol{M}^{*} \cdot \boldsymbol{v}\right\}_{n}^{\text {explicit }}+\left\{\boldsymbol{F}^{\text {int }}(\tilde{\boldsymbol{d}})\right\}^{\text {explicit }}-\boldsymbol{G}(\tilde{\boldsymbol{d}})
\end{array}\right.
$$

To obtain the macroscopic displacement and pore pressure at time $t_{n+1}$ from the non-linear equation system (43), Newton-Raphson iteration method is employed. Let us denote the corrected solutions as $d_{n+1}^{j}$ and $v_{n+1}^{j}$, at the time step $n+1$ and $j$ th iteration, i.e.,

$$
d_{n+1}^{j}=\tilde{d}+\alpha \Delta t v_{n+1}^{j}
$$

with $v_{n+1}^{0}=0$. The relationship of their increments is thus:

$$
\Delta d_{n+1}^{j}=\alpha \Delta t \Delta v_{n+1}^{j}
$$

The equation (43) in terms of these iterative solutions is written as:

$$
F_{\mathrm{IMP}}\left(v_{n+1}^{j+1}, d_{n+1}^{j+1}\right)+F_{\mathrm{EXP}}\left(v_{n+1}^{j}, d_{n+1}^{j}\right)=F_{n+1}^{e x t}
$$

The consistent linearization of the implicit part $\boldsymbol{F}_{\mathrm{IMP}}$ is required to solve (47). The resulting tangential stiffness matrix depends on what force terms are included in $\left\{\boldsymbol{M}^{*} \cdot \boldsymbol{v}\right\}^{\text {implicit }}$ and $\left\{\boldsymbol{F}^{\text {int }}\right\}^{\text {implicit }}$.

For the implicit-explicit split of the nonlinear rate of change term $\boldsymbol{M}^{*} \cdot \boldsymbol{v}$, note that, from (38) and (39), its variation contains two components:

$$
\frac{\partial\left(\boldsymbol{M}^{*} \cdot v\right)}{\partial v} \cdot \delta v=\left(\boldsymbol{M}^{*}+\frac{\partial \boldsymbol{M}^{*}}{\partial v} \cdot v\right) \cdot \delta v
$$


In the proposed scheme, the rate of change term is split in a way that only $M^{*}$ is treated implicitly:

$$
\frac{\partial\left(\boldsymbol{M}^{*} \cdot \boldsymbol{v}\right)}{\partial v}=\left\{\frac{\partial \boldsymbol{M}^{*} \cdot v}{\partial v}\right\}^{\text {implicit }}+\left\{\frac{\partial \boldsymbol{M}^{*} \cdot \boldsymbol{v}}{\partial v}\right\}^{\text {explicit }} ;\left\{\begin{array}{l}
\left\{\frac{\partial \boldsymbol{M}^{*} \cdot v}{\partial v}\right\}^{\text {implicit }}=M^{*} \\
\left\{\frac{\partial \boldsymbol{M}^{*} \cdot v}{\partial v}\right\}^{\text {explicit }}=\frac{\partial \boldsymbol{M}^{*}}{\partial v} \cdot \boldsymbol{v}
\end{array}\right.
$$

From the above implicit-explicit split, the first order linearization form of $\boldsymbol{F}_{\mathrm{IMP}}$ in (47) reads:

$$
\begin{aligned}
F_{\mathrm{IMP}}\left(v_{n+1}^{j+1}, d_{n+1}^{j+1}\right) & \simeq \boldsymbol{F}_{\mathrm{IMP}}\left(v_{n+1}^{j}, d_{n+1}^{j}\right)+M^{*} \cdot \Delta v_{n+1}^{j+1}+K_{T}^{\text {implicit }} \cdot \Delta d_{n+1}^{j+1} \\
& \simeq \boldsymbol{F}_{\mathrm{IMP}}\left(v_{n+1}^{j}, d_{n+1}^{j}\right)+\underbrace{\left[M^{*}+\alpha \Delta t K_{T}^{\text {implicit }}\right]}_{M^{* *}} \cdot \Delta v_{n+1}^{j+1}
\end{aligned}
$$

where

$$
K_{T}^{\text {implicit }} \cdot \Delta d_{n+1}^{j+1}=\left.\frac{d}{d \beta}\left\{F^{i n t}\left(d_{n+1}^{j}+\beta \Delta d_{n+1}^{j+1}\right)\right\}^{\text {implicit }}\right|_{\beta=0}
$$

is the directional derivative of $\left\{\boldsymbol{F}^{i n t}\right\}^{\text {implicit }}$ at $d_{n+\mathbf{1}}^{j}$ in the direction of $\Delta d_{n+\mathbf{1}}^{j+\mathbf{1}}$.

For construction of $K_{T}$ implicit firstly, a complete linearization of the internal force $F^{\text {int }}$ results in the following form of tangential stiffness matrix, according to (38) and (39):

$$
K_{T}=\frac{\partial F^{i n t}}{\partial d}=\left[\begin{array}{cc}
(\underbrace{K^{e}-K^{e p}}_{K^{s}}+K^{g e o}) & -K^{u p} \\
K_{1}^{p} & K^{p}
\end{array}\right]
$$

where $K^{e}$ is the elastic contribution and $K^{e p}$ is the non-linear elastic-plastic contribution to the material tangential stiffness $\boldsymbol{K}^{s}$. $\boldsymbol{K}^{g e o}$ represents the sum of the geometrical stiffness $\boldsymbol{K}_{S^{\prime}}^{g e o}$ and $\boldsymbol{K}_{p^{f}}^{\text {geo }}$.

Since computation of the homogenized $\boldsymbol{K}^{s}$ from DEM RVEs produces considerable computational cost, in the proposed multiscale solution scheme, we choose to treat $K^{e}$ implicitly and $\boldsymbol{K}^{e p}$ explicitly. $\boldsymbol{K}^{e}$ is thus evaluated at the initial time step using the elastic properties (bulk modulus $K_{b u l k}^{\mathrm{DEM}}$ and shear modulus $G_{\text {shear }}^{\text {DEM }}$ ) homogenized from the initial RVEs. $K^{u p}$ and $K^{p}$ are included in the implicit part of the tangential stiffness matrix. The geometrical terms $K^{g e o}$ and $K_{1}^{p}$ are treated explicitly. With these considerations and (52), the resulting operator split writes:

$$
\left\{\begin{aligned}
\boldsymbol{K}_{T} & =\boldsymbol{K}_{T}^{{ }^{\text {implicit }}+\boldsymbol{K}_{T}{ }^{\text {explicit }}} \\
\boldsymbol{K}_{T}{ }^{\text {implicit }} & =\frac{\partial\left\{\boldsymbol{F}^{\text {int }\}^{\text {implicit }}}\right.}{\partial d}=\left[\begin{array}{cc}
\boldsymbol{K}^{e}-\boldsymbol{K}^{u p} \\
\mathbf{0} & \boldsymbol{K}^{p}
\end{array}\right] \\
\boldsymbol{K}_{T}{ }^{\text {explicit }} & =\left[\begin{array}{cr}
-\boldsymbol{K}^{e p}+\boldsymbol{K}^{g e o} & 0 \\
\boldsymbol{K}_{\mathbf{1}}^{p} & 0
\end{array}\right]
\end{aligned}\right.
$$

From equations (44), (47), (49), (50) and (53), we obtain equation (54), which represents the iteration equation of the semi-implicit predictor-multicorrector scheme:

$$
M^{* *} \cdot \Delta v_{n+1}^{j+1}=\Delta F_{n+1}^{j}=F_{n+1}^{e x t}-M^{*} \cdot v_{n+1}^{j}-F^{i n t}\left(d_{n+1}^{j}\right)-G\left(d_{n+1}^{j}\right)
$$

where the internal force $\boldsymbol{F}^{i n t}\left(\boldsymbol{d}_{n+1}^{j}\right)$ has two contributions: the PK2 effective stresses which are homogenized from DEM RVEs and the macroscopic internal force from FEM, i.e.,

$$
F^{i n t}\left(d_{n+1}^{j}\right)=\left\{\begin{array}{c}
f^{i n t}\left(u_{n+1}^{j}\right) \\
0
\end{array}\right\}^{\mathrm{DEM}}+\left\{F^{i n t}\left(d_{n+1}^{j}\right)\right\}^{\mathrm{FEM}}
$$

$\phi^{\mathrm{DEM}}, B^{\mathrm{DEM}}, M^{\mathrm{DEM}}$ and $k^{\mathrm{DEM}}$ are homogenized at each time step to construct the tangential matrix $\boldsymbol{M}^{* *}$. The convergence is achieved when $\frac{\left\|\Delta F_{n+1}^{j}\right\|}{\left\|\Delta F_{n+1}^{0}\right\|} \leq$ TOL [Prevost, 1983]. In the numerical examples TOL is equal to $10^{-4}$. The matrix forms of the finite strain multiscale $u-p$ formulation are provided in the Appendix. To recapitulate and illustrate the multiscale semi-implicit scheme, we present a flowchart as shown in Fig.2. 


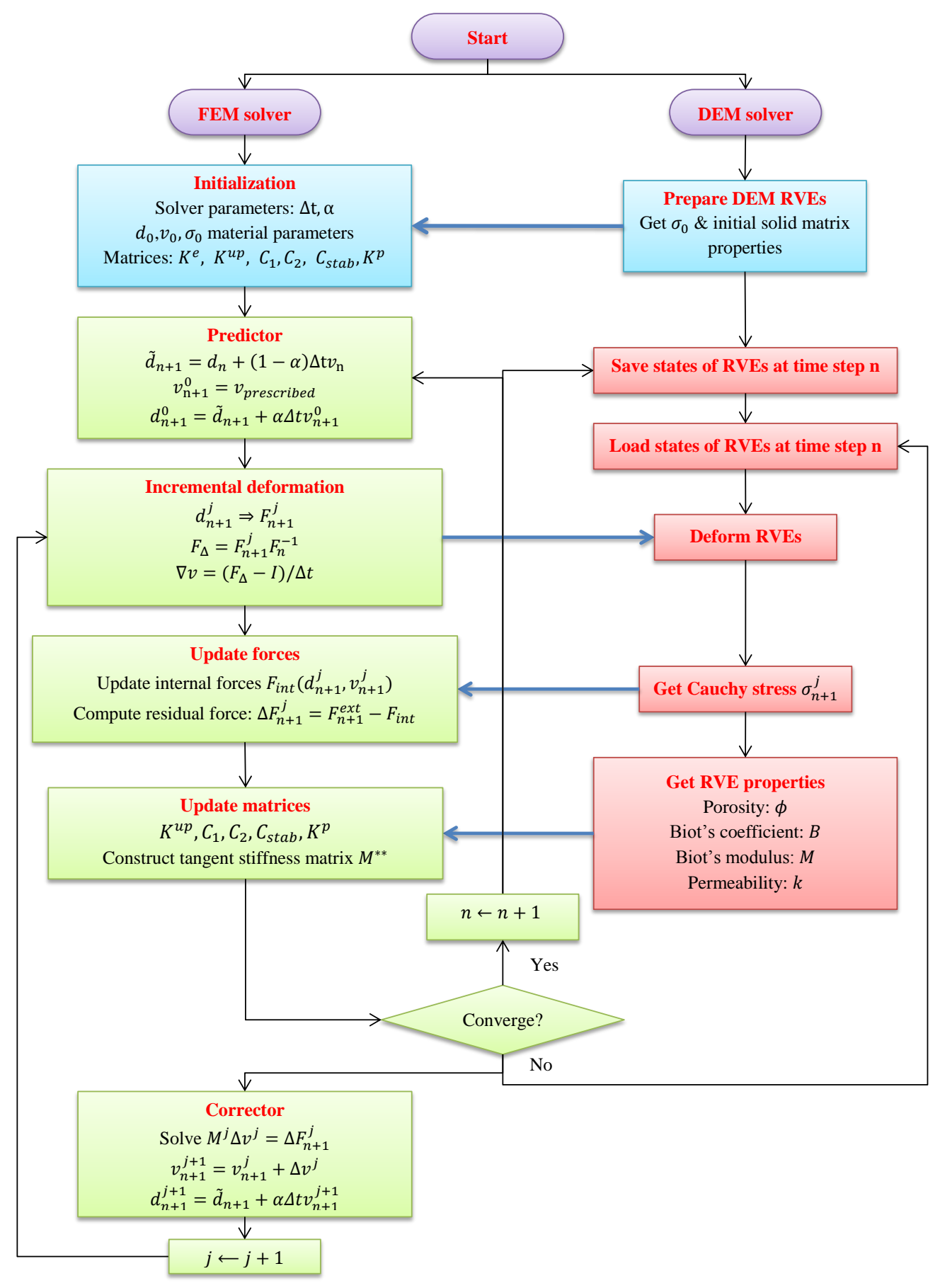

Fig. 2: Flowchart of the multiscale semi-implicit scheme. Blue blocks represent initialization steps of the solution scheme ; Green blocks refer to FEM solver steps and red blocks refer to DEM solver steps; Blue arrows indicate the information flow between the two solvers. 


\begin{tabular}{cc}
\hline Parameter & Value \\
\hline Solid matrix bulk modulus & $40 \mathrm{MPa}$ \\
Solid matrix shear modulus & $40 \mathrm{MPa}$ \\
Fluid bulk modulus & $22 \mathrm{GPa}$ \\
Permeability & $1 \times 10^{-9} \mathrm{~m}^{2} /(\mathrm{Pa} \cdot \mathrm{s})$ \\
Solid density & $2700 \mathrm{~kg} / \mathrm{m}^{3}$ \\
Fluid density & $1000 \mathrm{~kg} / \mathrm{m}^{3}$ \\
Porosity & 0.375 \\
\hline
\end{tabular}

Table 1: Material parameters in Terzaghi's problem

\section{Numerical Examples}

The objective of this section is to demonstrate the versatility and accuracy of the proposed method in both the small and finite deformation ranges. The numerical examples in this section are the representative problems commonly encountered in geotechnical engineering. The first example is the Terzaghi's problem, which serves as a benchmark to verify the implementation of the numerical schemes proposed in this paper. This example is followed by a globally undrained shear test which examines how granular motion altered by fluid seepage within a soil specimen affects the macroscopic responses. In the third example, we simulate the responses of a cylindrical DEM-FEM model with drained condition subjected to triaxial compression loading with both quarter- and full domains and found that the quarter simulation may suppress the non-symmetric bifurcation mode that leads to shear band. The analysis on fabric tensor also reveals that the fabric and deviatoric stress tensors are almost co-axial inside the shear band, but they are not co-axial in the host matrix. The last example is a slope stability problem in which only a portion of domain, i.e. the slip surface is modeled by the DEM-FEM model and compared with the prediction done via SLOPE/W. The result shows that the multiscale analysis may lead a more conservative prediction than the classical Bishop's method.

\subsection{Verification with Terzaghi's one-dimensional consolidation}

We verify our semi-implicit DEM-mixed-FEM scheme in both infinitesimal strain and finite strain regimes with the Terzaghi's 1D consolidation problem. This benchmark problem serves two purposes. First, we want to ensure that the semi-implicit FE algorithm converges to the analytical solution in the geometrically linear regime. Second, we want to assess whether the geometrical effort is properly incorporated in the numerical scheme in the finite deformation range. The Terzaghi's problem is well known and exact solution is available [Terzaghi et al., 1943]. While numerical solution of Terzaghi's problem does not provide much new insight beyond the established results, comparing the numerical solution with the analytical counterpart is nevertheless an important step in the verification procedure. In particular, this comparison ensures that the implementation of the model accurately represents the conceptual description and specification, as pointed out by Jeremić et al. [2008].

The model consists of a soil column of $10 \mathrm{~m}$ deep discretized by 10 3D hexahedral finite elements of size $1 \mathrm{~m}$ each along the column. The bottom surface is fixed and undrained, while the top boundary is drained and subjected to pressure of $1 \mathrm{MPa}$ (small strain condition) and $10 \mathrm{MPa}$ (large strain condition). The lateral surfaces are all impermeable and allow only vertical displacements. The material parameters assumed in this example are recapitulated in Table 1. Firstly the simulation is performed under consolidation pressure of $1 \mathrm{MPa}$ from $0 \mathrm{~s}$ to $500 \mathrm{~s}$ with time step of $1 \mathrm{~s}$. The comparison between the analytical solution and the numerical solution shown in Fig. 3 verifies the correctness of the u-p semi-implicit scheme and the effectiveness of the fluid pressure Laplacian stabilization scheme.

To illustrate the influence of geometrical non-linearity and varied permeability on the consolidation behavior, the soil column is subjected to a pressure of $10 \mathrm{MPa}$, resulting in a final vertical strain of about $8 \%$. The pressure and displacement evolution computed by different formulations are shown in Fig. 4 . The 


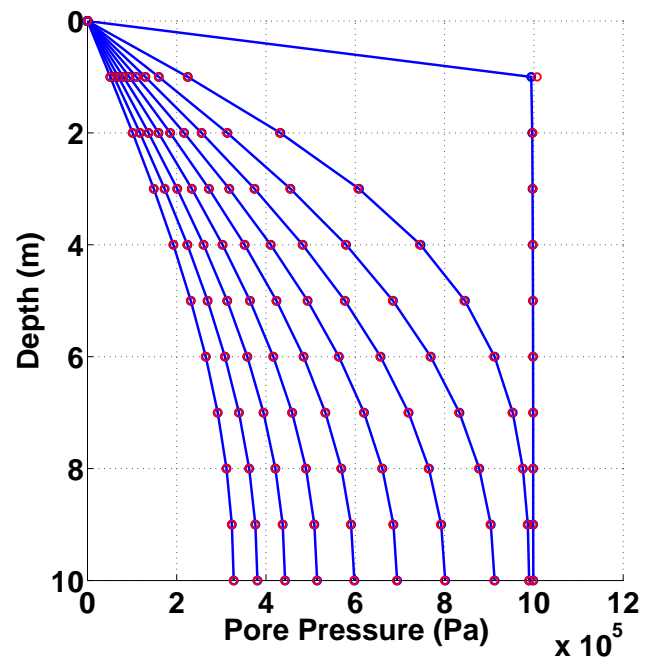

(a)

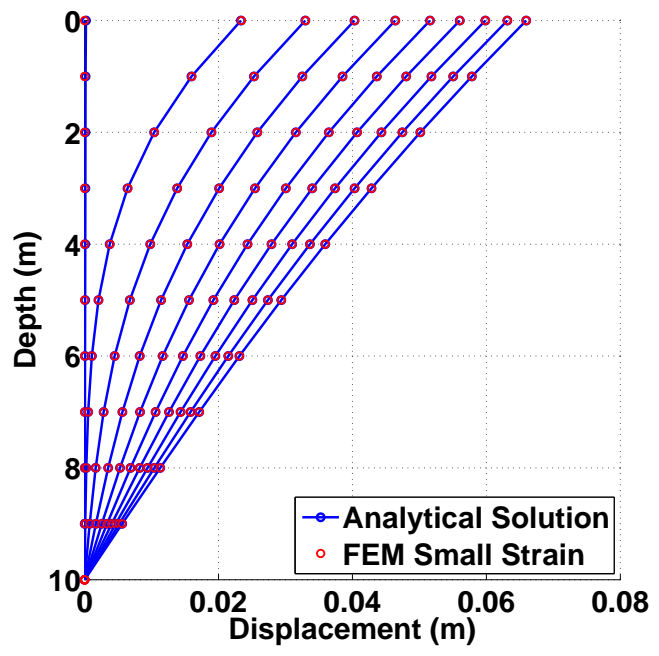

(b)

Fig. 3: Response of saturated soil column under $1 \mathrm{MPa}$ consolidation pressure from $0 \mathrm{~s}$ to $500 \mathrm{~s}(50 \mathrm{~s}$ between adjacent lines), verification of small strain formulation with analytical solutions (a) Pore pressure (b) Vertical displacement

finite strain scheme with constant permeability and the small strain scheme yield the same results until a compression of about $5 \%$ vertical strain (at $250 \mathrm{~s}$ ). Thereafter, due to the additional geometric nonlinear terms, the finite strain scheme gives smaller displacement and pressure compared to small strain, indicating that geometrical non-linearity becomes significant. When the permeability is permitted to decrease along with the reduced porosity during consolidation according to the Kozeny-Carman relation, the soil column requires more time to reach the final steady state, yet the final values remain the same as finite strain with constant permeability. The above observations are consistent with previous numerical studies [Li et al., 2004, Regueiro and Ebrahimi, 2010] .

\subsection{Globally undrained shear test of dense and loose assemblies}

For the second example we employ our multiscale scheme to perform shear tests on both dense and loose granular assemblies. The macroscopic geometry and boundary conditions are illustrated on a sample discretized by coarse mesh $(1 \times 5 \times 5$ in $X, Y, Z$ directions $)$ as Fig. 5 . We also use a medium fine mesh $(1 \times 8 \times 8)$ and a fine mesh $(1 \times 10 \times 10)$ to investigate the mesh dependency issue of the proposed scheme. All results in this section are computed from the fine mesh model, if not specified. The nodes on the bottom boundary are fixed in all directions and those on the upper boundary are translated identically towards the positive $\mathrm{y}$ axis at a constant rate. They are maintained at a constant vertical stress $\sigma_{z}=100 \mathrm{kPa}$ by a horizontal rigid layer (not shown). This constraint is imposed in the model by the Lagrange multiplier method. The lateral surfaces are constrained by frictionless rigid walls (not shown). All surfaces are impervious. The gravitational effect is not considered in this study. For coupled microscopic DEM models, periodic unit cells composed of uniform spheres are prepared by an isotropic compression engine in YADE up to $\sigma_{i s o}=100 \mathrm{kPa}$ with initial porosity of 0.375 and 0.427 for dense and loose assemblies respectively, and then are assigned identically to all the integration points of the FEM model before shearing.

The finite strain formulation is first adopted to study the hydro-mechanical coupling effect during the shearing of the dense and loose samples with undrained boundaries. The material parameters used in the simulations allowing hydraulic diffusion within the specimen are presented in Table 2 . They are categorized into micromechanical material parameters used in DEM solver, poro and poro-plasticity parameters 


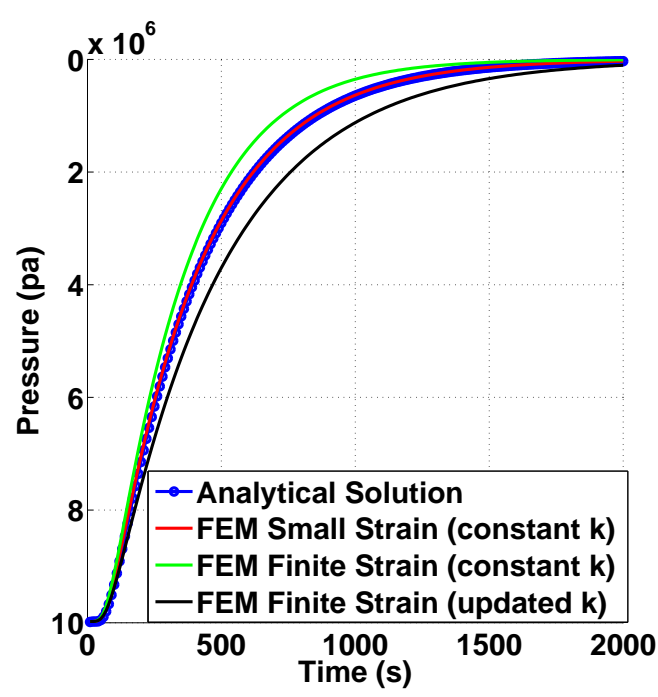

(a)

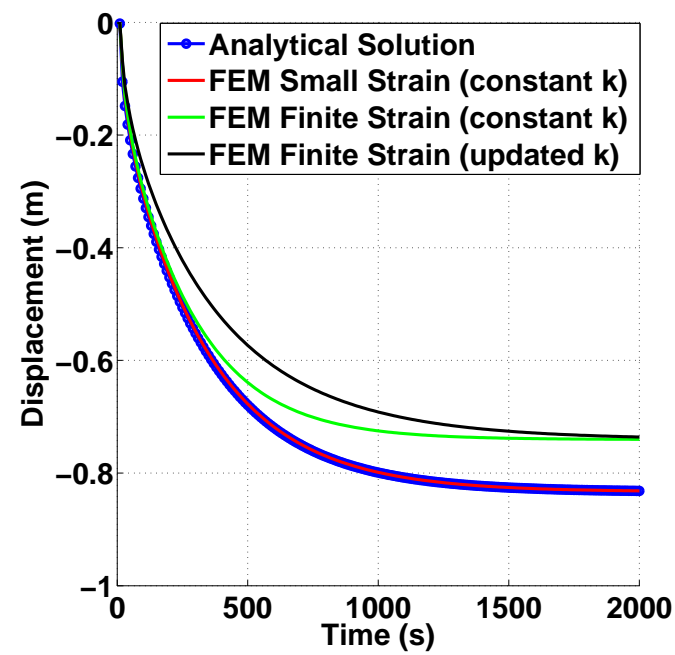

(b)

Fig. 4: Response of saturated soil column under $10 \mathrm{MPa}$ consolidation pressure. Comparison of analytical solution, numerical result from formulations of small strain, large strain with constant permeability $\mathrm{k}$ (in $\mathrm{m} / \mathrm{s}$ ) and large strain with $\mathrm{k}$ updated by the Kozeny-Carman equation. (a) Pore pressure evolution at bottom surface (b) Vertical displacement evolution at top surface

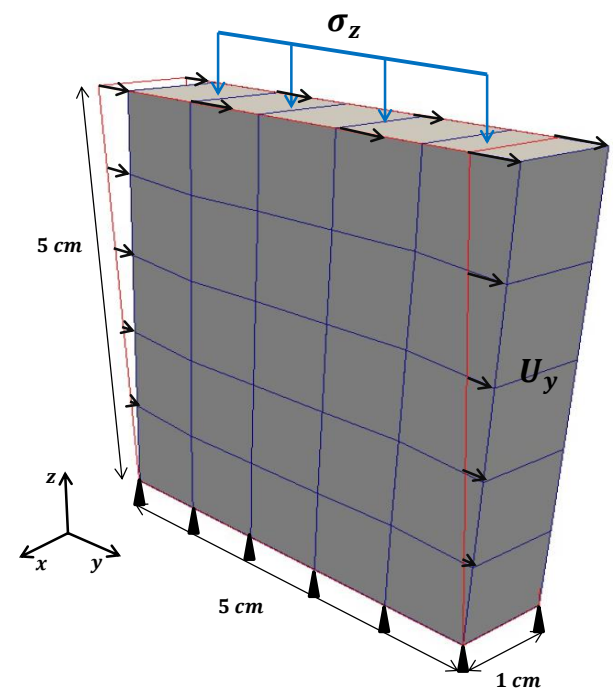

Fig. 5: Geometry and boundary conditions for globally undrained shear test

derived from DEM RVEs and macroscopic properties set in FEM. Note that the permeability $k$ is updated with porosity of RVEs using the Kozeny-Carman relation during the simulation. To prevent local seepage of water within the samples, the permeability $k$ is set to $0 \mathrm{~m}^{2} /(\mathrm{Pa} \cdot \mathrm{s})$. 


\begin{tabular}{ccc}
\hline & Parameter & Value \\
\hline Microscopic property & Solid grain normal stiffness $k_{n}$ & $2.2 \times 10^{6} \mathrm{~N} / \mathrm{m}$ \\
in DEM & Solid grain tangential stiffness $k_{s}$ & $1.9 \times 10^{6} \mathrm{~N} / \mathrm{m}$ \\
& Solid grain friction angle $\beta$ & $30^{\circ}$ \\
& Solid grain bulk modulus $K_{s}$ & $0.33 \mathrm{GPa}$ \\
\hline $\begin{array}{c}\text { Macroscopic property } \\
\text { inferred from DEM }\end{array}$ & Porosity $\phi$ & dense: 0.375, loose: 0.427 \\
& Biot's coefficient $B$ & dense: 0.976, loose: 0.983 \\
\hline Macroscopic property & Biot's Modulus $M$ & dense: $180 \mathrm{Mpa}$ loose: $168 \mathrm{Mpa}$ \\
in FEM & Fluid bulk modulus $K_{f}$ & $0.1 \mathrm{GPa}$ \\
& Initial permeability $k$ & $1 \times 10^{-9} \mathrm{~m}^{2} /(\mathrm{Pa} \cdot \mathrm{s})$ \\
& Solid density $\rho_{s}$ & $2700 \mathrm{~kg} / \mathrm{m}^{3}$ \\
& Fluid density $\rho_{f}$ & $1000 \mathrm{~kg} / \mathrm{m}^{3}$ \\
\hline
\end{tabular}

Table 2: Material parameters in globally undrained shear problem

Fig. 6 represents the global shear stress and volumetric strain behavior of shear simulations with and without local seepage of water. The strain-hardening behavior of undrained dense granular assemblies (left column) and strain-softening behavior of undrained loose granular assemblies (right column) are recovered [Yoshimine et al., 1998]. In both assemblies, when local seepage is prohibited, the shear stress immediately rises when the shearing begins and the saturated porous media behaves stiffer than the samples with local seepage. Note that the sudden drop in Fig. 6(b) is due to the unstable solid matrix of loosely confined DEM unit cell. The volumetric strain of the dense sample with seepage monotonically increases. This phenomenon is attributed to the rearrangement of solid matrix as the grains tend to rise over adjacent grains when they are driven by shear forces. In absence of local diffusion, the dense sample experiences a reduction of volume instead, suggesting that the compression of overall solid matrix predominates the above phenomenon. As for loose samples, however, the volumetric behavior is opposite. When local diffusion of water is prohibited, the pore collapse and densification of local regions within specimen could occur, resulting in a compression at early stage of shearing before the dilatancy phenomenon. The curve of no-local-seepage case shows that the dilatancy phenomenon prevails all along the shearing. In all cases, the volume changes are beneath $0.12 \%$, confirming that the samples are indeed sheared under globally undrained condition.

We examine the mesh dependency by three aforementioned mesh densities adopted in simulations of dense assembly with local seepage. The effect is presented via plots of global $\sigma_{y z}-\gamma_{y z}$ and $\varepsilon_{v}-\gamma_{y z}$ responses as Fig. 7. For stress response, discrepancy between medium and fine meshes is not significant, but coarse mesh apparently yields stiffer solution after $2 \%$ shear strain and the maximum deviation is about $7.6 \%$ with respect to the fine mesh solution. The differences between $\varepsilon_{v}$ curves are less significant and do not exceed $4 \%$ of the fine mesh solution. Thus, our choice of the fine mesh to conduct numerical experiments is acceptable.

We next display the difference between finite and small strain multiscale schemes for simulations of dense granular sample in both local diffusion conditions in Fig. 8. According to the global shear responses, the small strain and finite strain yield consistent solutions within $2 \%$ shear strain. Then the discrepancy gradually emerges and the introduction of geometrical non-linearity renders the sample stiffer. This observation is the same as the conclusion in the previous Terzaghi's problem section. Finite strain solutions exhibit less volume changes in both cases. Moreover, geometrical non-linear term even alters the dilatancy behavior: the sample is computed to be compressed when no local seepage of water is allowed, while the small strain solution conserves the dilatant trend.

We also assess the local diffusion effect via color maps of pore pressure developed during the deformation, as shown in Fig. 9. The dense sample with local seepage has developed negative pore pressure and the pressure distribution is nearly uniform, since fluid flow could take place inside the specimen to dissipate pressure difference between neighboring pores. Without local seepage of water, the pore pressure is concentrated to four corners of the sample, with the upper left and bottom right corners compressed (pos- 


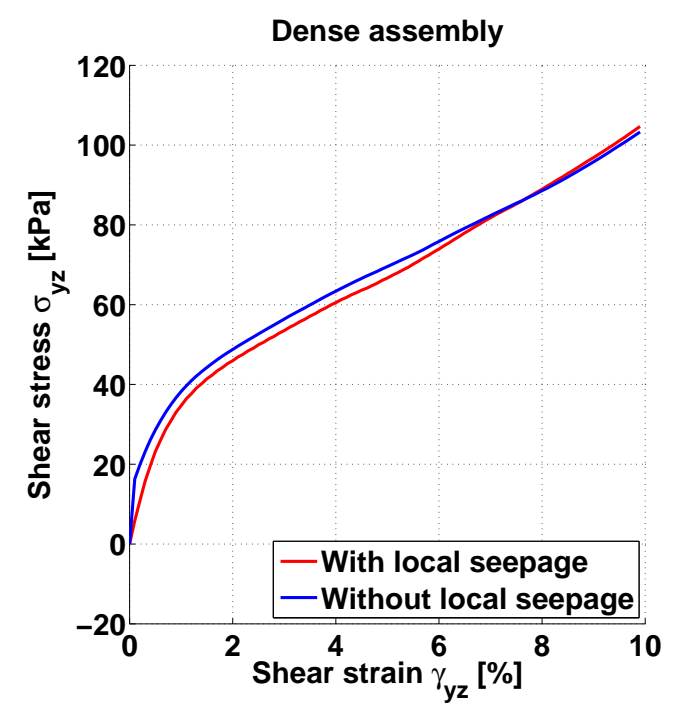

(a)

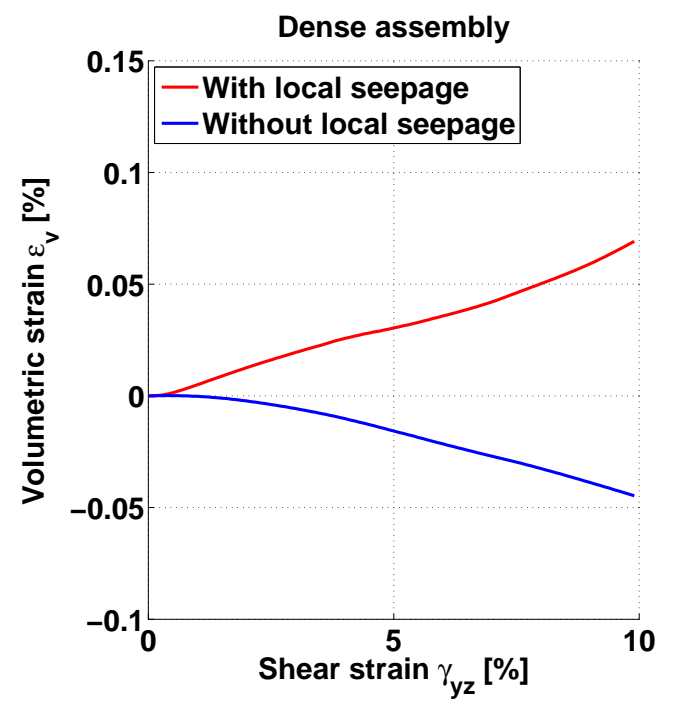

(c)

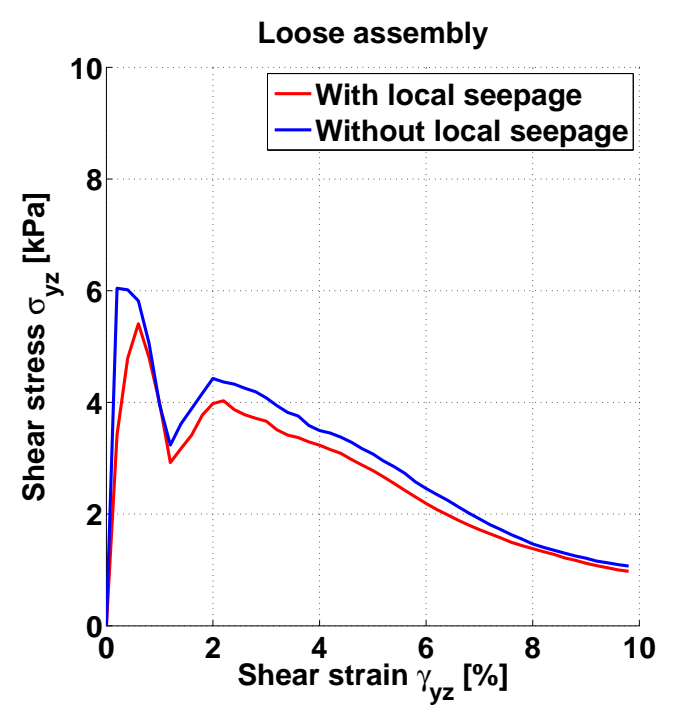

(b)

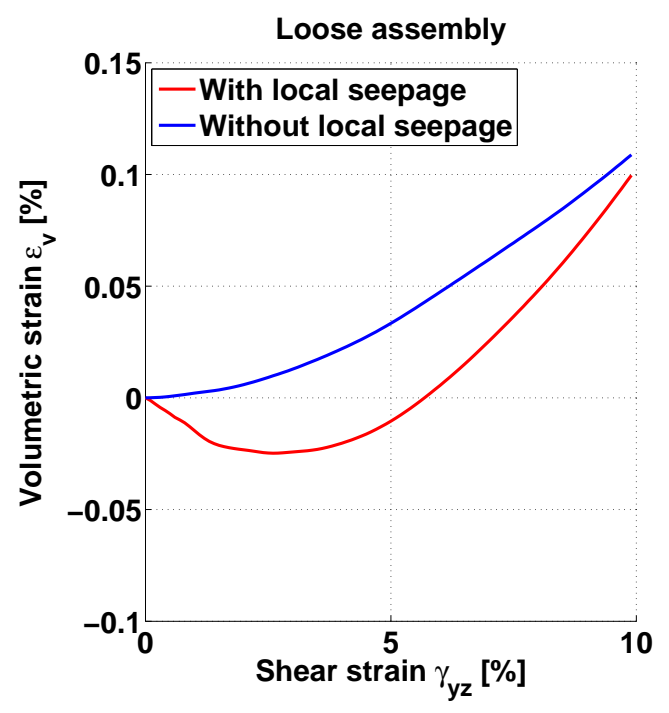

(d)

Fig. 6: Comparison of global shear stress and volumetric strain behavior between globally undrained dense and loose assemblies with and without local diffusion

itive pressure) and the other two dilated (negative pressure). Furthermore, these corners have maximum pressure gradient $\left\|\nabla p^{f}\right\|$.

The multiscale nature of our method offers more insight into the local states of granular sample. With the granular material behavior homogenized from responses of RVEs, the grain displacements, the effective stress paths (shear stress $q=\sigma_{1}-\sigma_{3}$ vs. effective mean stress $p^{\prime}=\frac{\sigma_{1}+\sigma_{2}+\sigma_{3}}{3}$ ) and the volumetric strain paths $\left(\varepsilon_{v}\right.$ vs. $\left.p^{\prime}\right)$ in each DEM unit cell are directly accessible. As an example, the local distribution of $q$ at the end of shearing for globally undrained yet locally diffused dense sample (10) shows a concentration of shear stress in upper left and bottom right corners, while the corners correspondent to the other diagonal sustain comparably very little shear stress. The deformed configuration of spheres in three representative RVEs are colored according to the dimensionless displacement magnitude $\frac{\|\boldsymbol{u}\|_{2}}{\text { inital size of unit cell }}$ compared to initial 


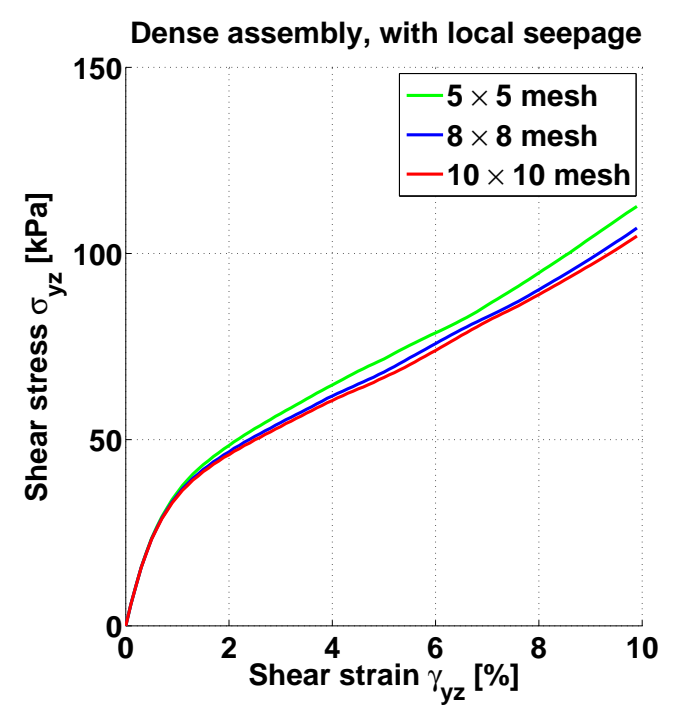

(a)

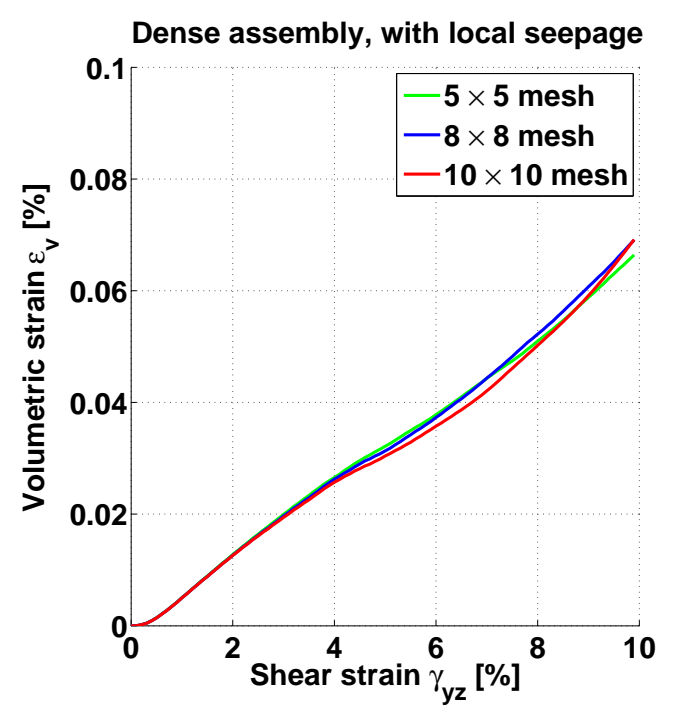

(b)

Fig. 7: Comparison of global shear stress and global volumetric strain behavior between coarse mesh $(1 \times 5 \times 5)$, medium mesh $(1 \times 8 \times 8)$, fine mesh $(1 \times 10 \times 10)$, finite strain formulation

RVE configuration. We present stress paths of these three RVEs providing evidence that strain-softening (Fig. 11(a)), limited strain-softening (Fig. 11(b)) and strain-hardening (Fig. 11(c)) could locally occur in a dense sample which globally behaves in a strain-hardening manner. A critical state line $q=\eta p^{\prime}$ is drawn for three stress paths and the value of slope $\eta$ is identified as 1.16. $\eta$ and the Mohr-Coulomb friction angle $\beta^{\prime}$ is computed to be $29.1^{\circ}$ by the following relation for cohesionless soil [Wood, 1990]:

$$
\sin \beta^{\prime}=\frac{3 \eta}{6+\eta}
$$

, which is close to the inter-particle friction angle $\beta=30^{\circ}$. Paths of $\varepsilon_{v}$ further demonstrate that large local volume change up to $5.5 \%$ is possible even globally the sample is only dilated about $0.07 \%$. According to these figures, the small strain and finite strain shear responses are almost identical. The stress path curves exhibit little difference. However, geometrical non-linearity has more significant effect on volumetric strain path. A major remark is that, inside the strain-softening spot as 11(d), the small strain solution has large fluctuation when the mean effective stress is very small, because DEM assemblies are highly unstable with nearly zero confining stress. On the contrary, finite strain scheme avoids this unstable regime and yield smooth solutions.

Lastly, we investigate the rate-dependent shearing behavior using the proposed coupling scheme. A faster shearing of saturated granular sample influences its mechanical response mainly by speeding up the solid matrix re-arrangement and also by allowing less fluid diffusion inside the sample between loading steps. The former effect leads to swelling of the sample, while the latter renders the specimen more locally undrained. Fig.12 illustrates the combined effect of these two mechanisms on a dense sample with local seepage. The evolution of shear stress and volumetric strain with shearing rates of $0.1 \%$ and $0.5 \%$ per second are compared. When shearing is completed, shear stress sustained by the sample increases about $4.6 \%$ under higher shearing rate. The rate effect on volumetric strain is more prominent, by the fact that the sample experiences more volume expansion of about $13.5 \%$ at the end.

\subsection{Globally drained triaxial compression test}

The third example consists of the globally drained triaxial compression test on an isotropically consolidated cylindrical specimen. This example demonstrates the applicability of the proposed multiscale finite strain 


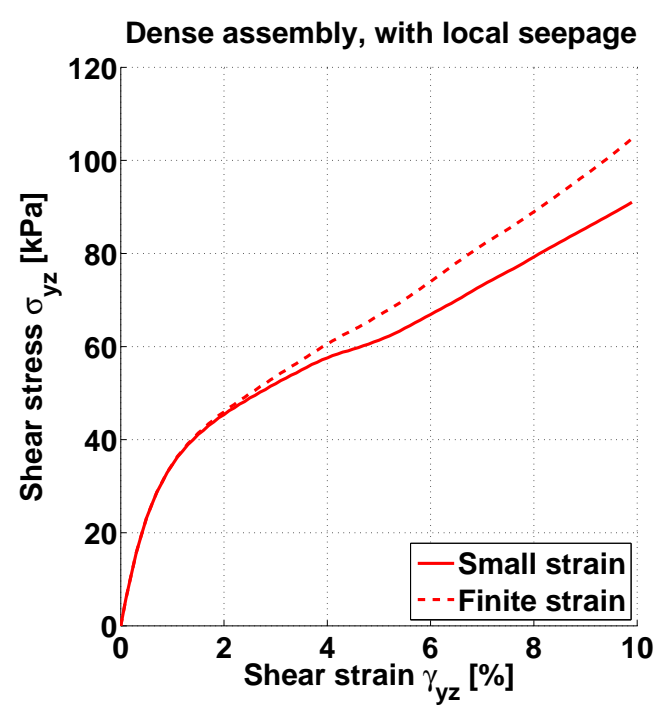

(a)

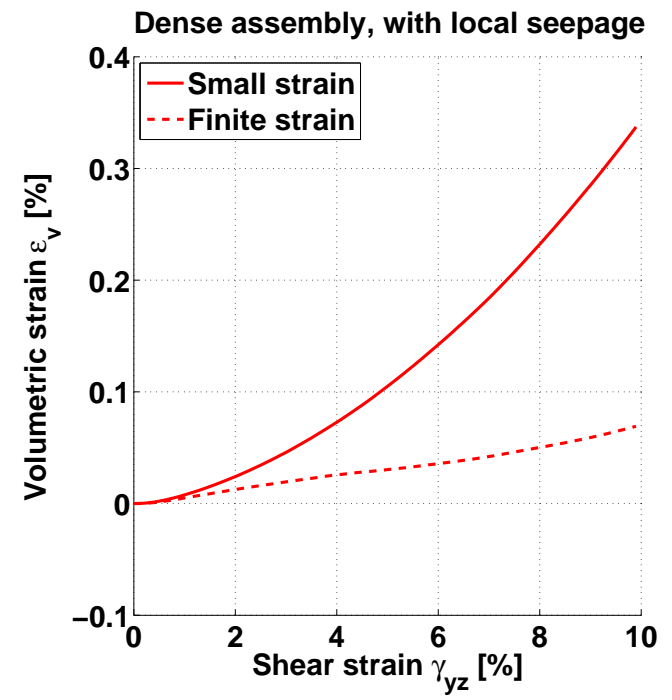

(c)

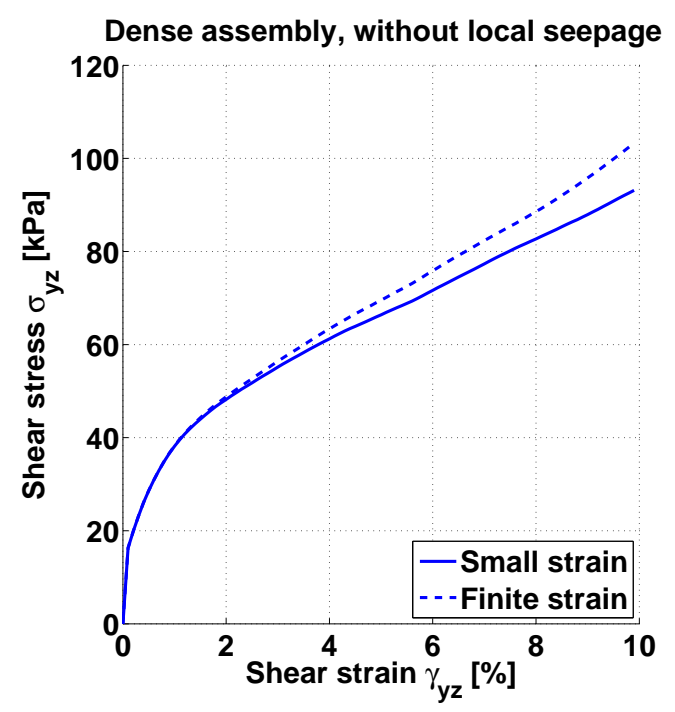

(b)

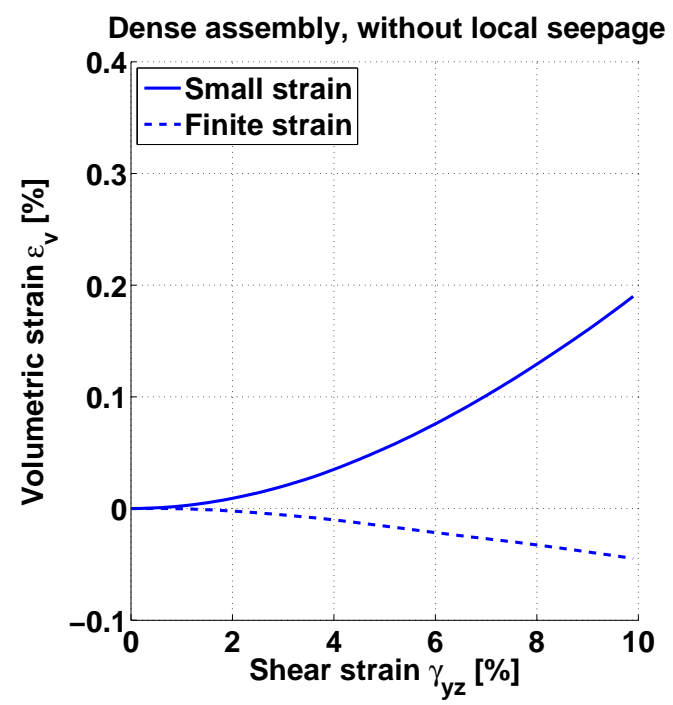

(d)

Fig. 8: Comparison of global shear stress and global volumetric strain behavior between small strain and finite strain formulation. Left: globally undrained with local diffusion condition, Right: globally undrained but without local diffusion condition

scheme on 3D problems. In this numerical example, we analyze (1) the difference between quarter-domain and full-domain simulations for material subjected to axial-symmetrical loading, (2) the consequence of the build-up of excess pore pressure due to a high loading rate and (3) the evolution of the fabric tensor inside and outside the shear band and the implications on the critical state of the materials. As a result, water is allowed to flow through the bottom and the top of the specimen. However, triaxial compression simulation is intentionally not conducted under a fully drained condition at a material point level. Instead, the rate dependence of the constitutive responses introduced via the hydro-mechanical coupling effect is studied to quantify what is the acceptable range of the prescribed loading rate that can prevent significant amount of excess pore pressure. 


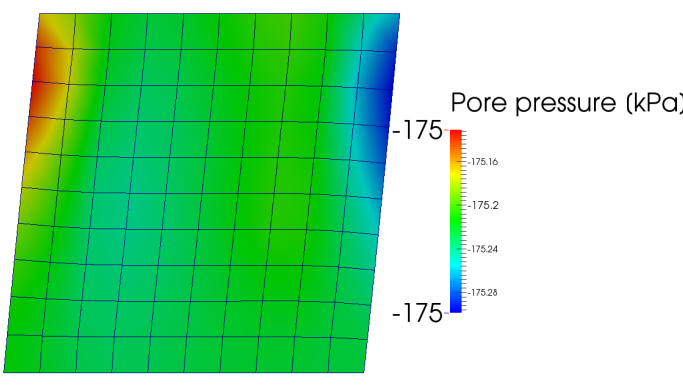

(a)

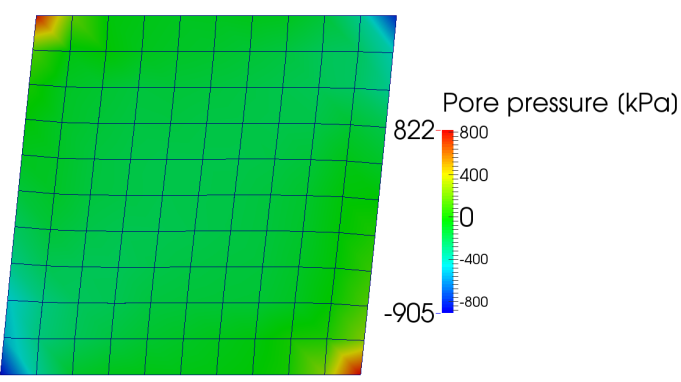

(b)

Fig. 9: Comparison of pore pressure at 10\% shear strain between (a) dense sample with local seepage and (b) dense sample without local seepage

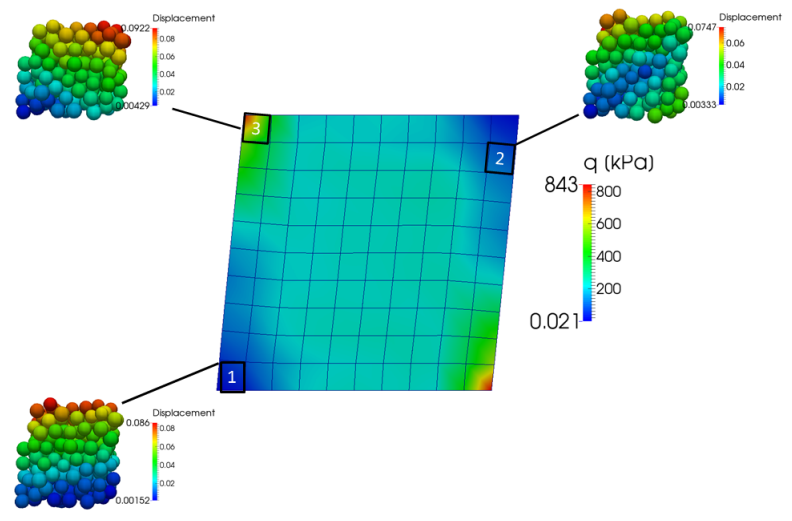

Fig. 10: Spatial distribution of shear stress $q$ at $10 \%$ shear strain for globally undrained dense sample allowing seepage within the specimen, attached with displacement magnitude of grains in unit cells (normalized by the initial cell size)

In addition, microscopic information such as the Biot's coefficient, Biot's modulus and micro-structure fabric are provided to highlight the advantage of the DEM-FEM coupled model. The convergence profile of this simulation is also presented. In an experimental setting, the drained triaxial test is performed on a cylindrical water-saturated soil specimen, laterally enveloped by rubber membrane and drained through top and bottom surfaces. One of the idealized 3D numerical model constitutes only a quarter of the cylinder by assuming the rotational symmetry. The constant confining pressure is directly applied on the lateral surface, neglecting the effect of rubber membrane. The quasi-static compression is achieved by gradually increasing the axial strain $\varepsilon_{z}$ at the rate of $0.05 \%$ per second. The lateral surface is impermeable and a no-flux boundary condition is imposed, while the pore water pressure on both top and bottom surfaces are constrained to be 0 . Another simulation is triaxial compression of the full cylindrical domain. Similar confining pressure and pore pressure boundary conditions are applied. The middle point of the bottom surface is fixed to prohibit rigid body translation. The geometry, mesh and boundary conditions of the quarter-/full-domain simulations are illustrated in Fig. 13. The DEM assembly adopted in these simulations is identical to the dense sample in the previous section. The fluid bulk modulus in this example is 2.2 GPa.

Fig. 14 compares the global shear stress and volumetric strain behavior from quarter-domain and full domain simulations. The shear stress curve obtained from full-domain simulation exhibits less peak stress 


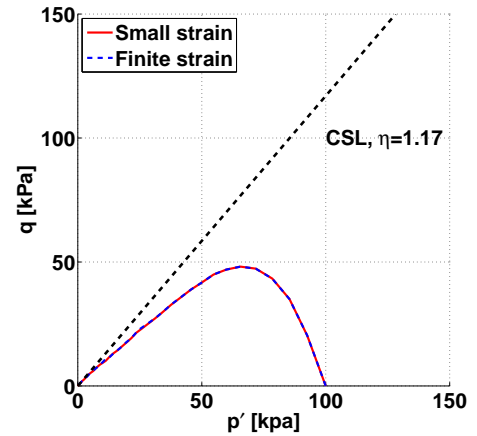

(a)

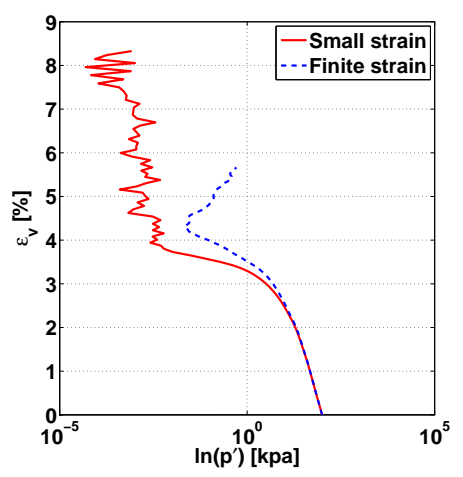

(d)

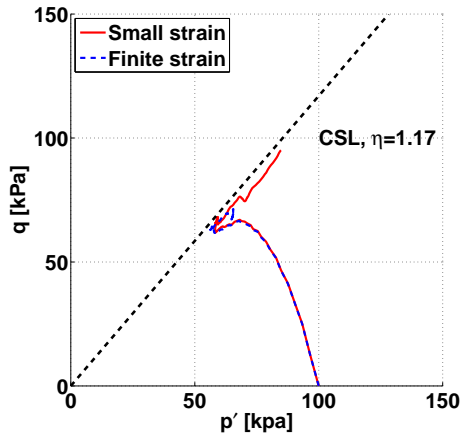

(b)

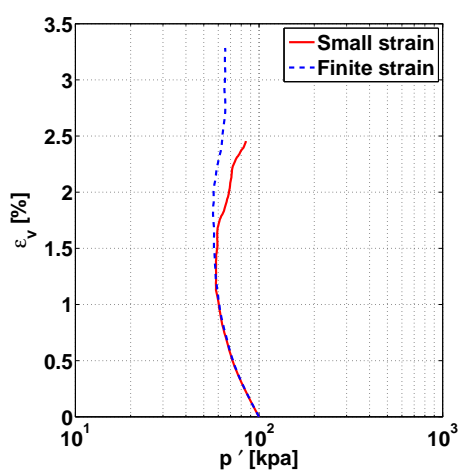

(e)

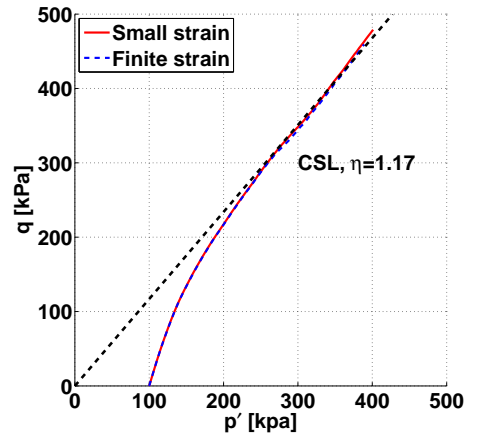

(c)

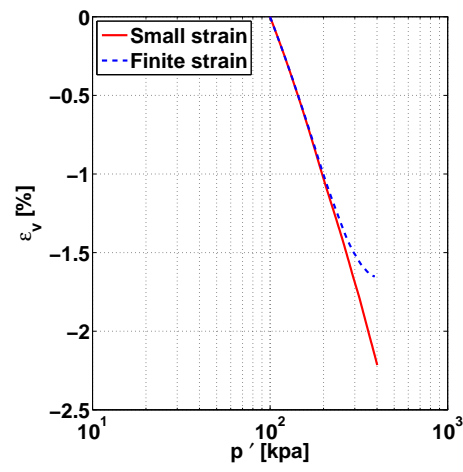

(f)

Fig. 11: Shear stress vs. effective mean stress at different locations indexed as Fig. 10: (b) stress path at point 1 (c) stress path at point 2 (d) stress path at point 3; Volumetric strain vs. effective mean stress at different locations: (e) volume path at point 1 (f) volume path at point 2 (g) volume path at point 3

and more significant softening than quarter-domain simulation. The volumetric strain curves, however, only show notable difference after the axial strain approaches $7 \%$. This discrepancy may be attributed to the strain localization in full-domain simulation, as shown by the distribution of deviatoric strain and porosity in Fig. 15. A dilatant shear band is developed inside the cylindrical specimen, while in the quarterdomain, the deformation is nearly homogeneous. This difference is more profound given the fact that the proposed model also incorporates the geometrical effect at the finite strain range. Results from this set of simulations show that the quarter-domain simulation is insufficient to capture the deformed configuration when bifurcation occurs. While the assumption of axial-symmetry is valid before the onset of strain localization, enforcing axial-symmetry via reduced domain and additional essential boundary condition may eliminate the bifurcation mode(s) that is not axial-symmetric.

An additional full-domain simulation is performed at a strain rate ten times slower: $\dot{\epsilon}_{z}=0.005 \%$ per second. The global shear stress and volumetric strain behavior are compared for the two loading rates in Fig. 16. The specimen under higher strain rate can sustain higher shear stress, but the strain rate has very little influence on volumetric strain behavior. The evolution of pore pressure at the center of the cylindrical specimen in two cases are also shown in Fig. 17. At a high strain rate, the pore water does not have time to fully diffuse through local pores and reach steady state. As a result, excess pore pressure builds up to about $5 \mathrm{kPa}$ while the specimen shrinks. The pressure then decreases and becomes negative when the specimen dilates. In the low-strain-rate case, the magnitude of pore pressure is about five times smaller while the trend looks similar of the high-strain-rate counterpart. 


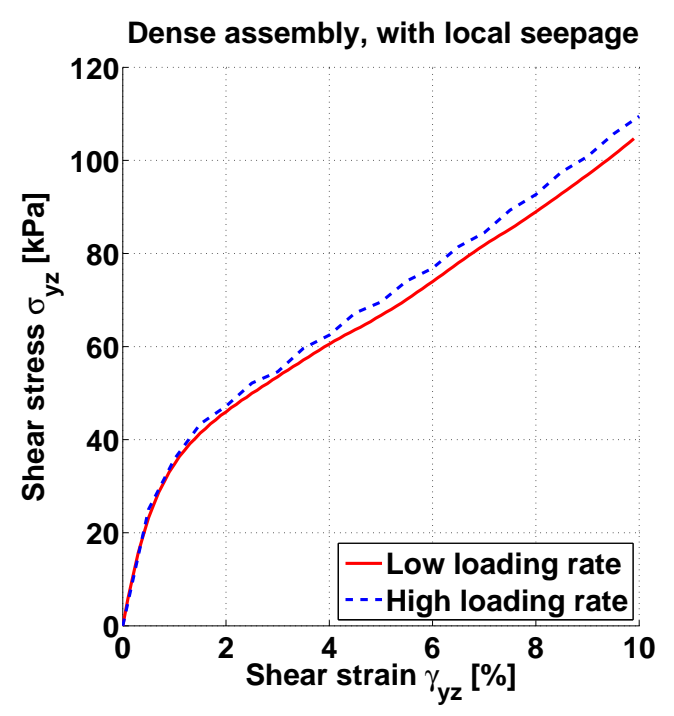

(a)

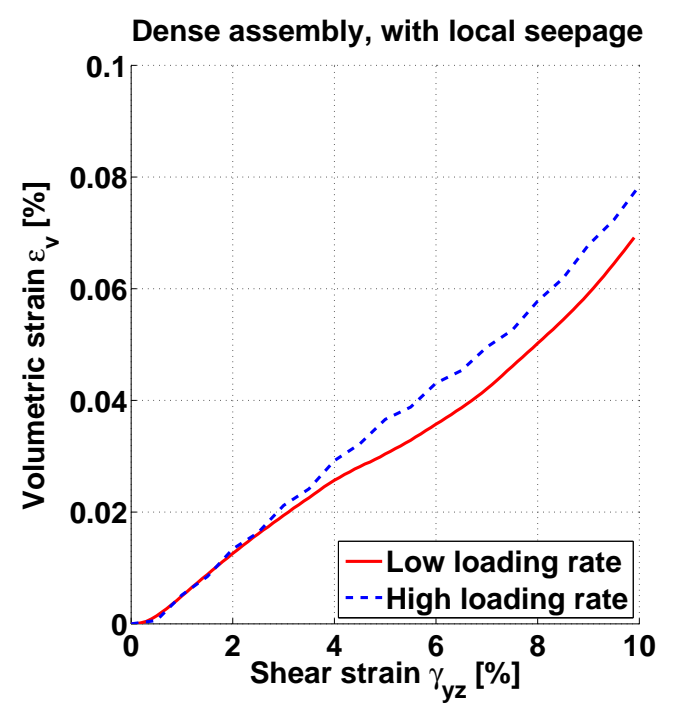

(b)

Fig. 12: Comparison of global shear stress and global volumetric strain behavior between low loading rate $(0.1 \%$ shear strain per second) and high loading rate $(0.5 \%$ shear strain per second), finite strain formulation

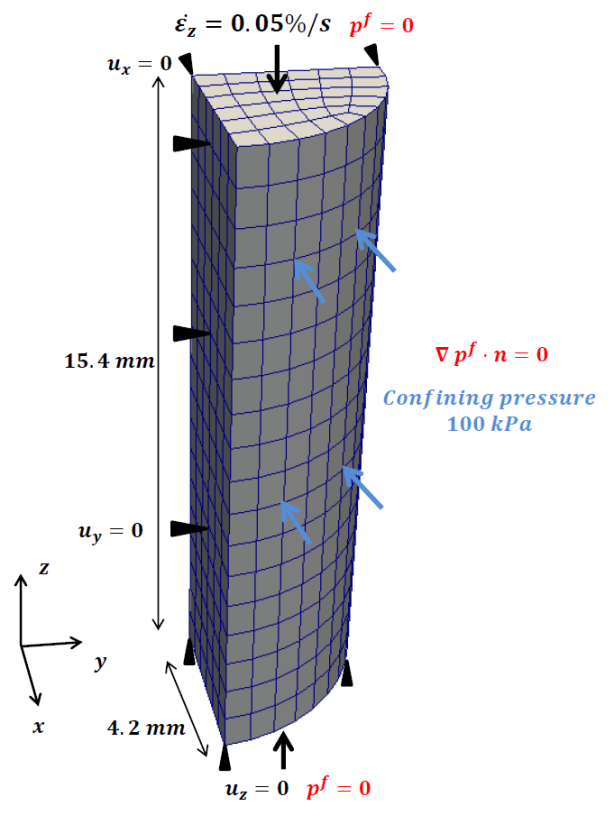

(a)

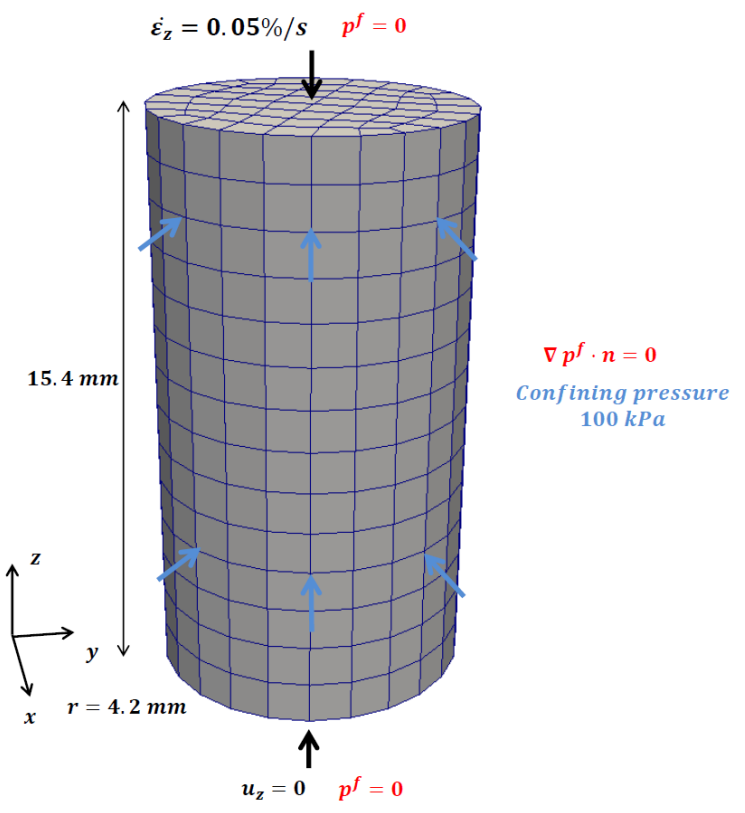

(b)

Fig. 13: Geometry, mesh and boundary conditions for globally drained triaxial compression test. (a) Quarter-domain simulation. (b) Full-domain simulation

One of the advantages of substituting macroscopic phenomenological constitutive model with DEM simulations for the poromechanics problem is that the macroscopic poro-elasticity properties, such as Biot's coefficient $B$, Biot's modulus $M$ and effective permeability $k$ could be inferred and updated from DEM at each Gauss point. As a result, the spatial variability of these poro-elasticity parameters triggered by 


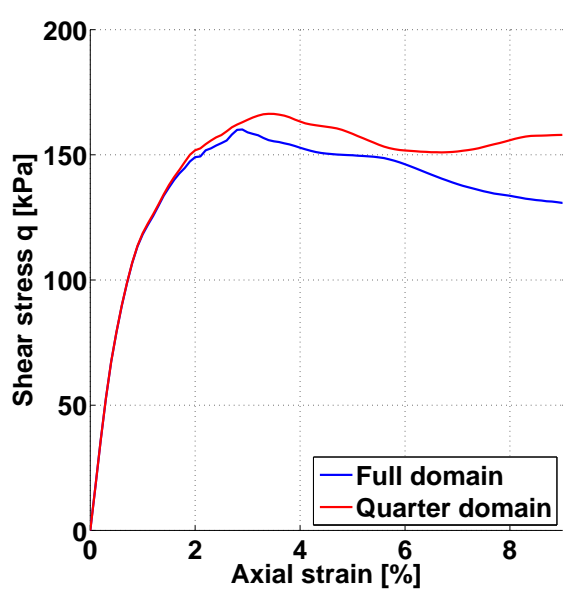

(a)

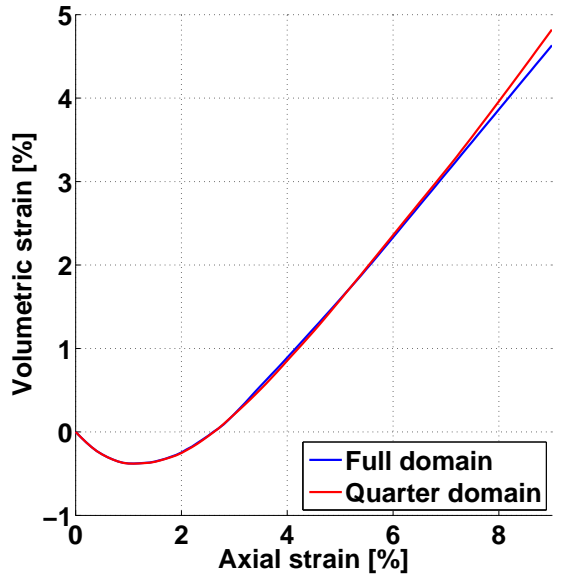

(b)

Fig. 14: Global shear stress and volumetric strain behavior in globally drained triaxial compression test. Comparison of quarter-domain and full-domain simulations

material bifurcation or non-homogeneous loading can be properly captured. As an example, we monitor the evolution of these poro-elasticity parameters against axial strain $\varepsilon_{z}$ for a RVE inside the shear band (RVE A, shown in Fig. 15(c)) and another RVE outside the shear band (RVE B, shown in Fig. 15(c)) in the $\dot{\epsilon_{z}}=0.05 \%$-per-second, full-domain simulation (Fig. 18). The evolution of the Biot's coefficient $B$ shown in Fig. 18)(a) suggests that the effective bulk modulus of the solid skeleton $\left(K_{T}^{\mathrm{DEM}}\right)$ first increases and then decreases presumably due to the porosity changes in both RVEs A and B. The Biot's modulus $M$, which is related to the Biot's coefficient $B$ and porosity $\phi$, exhibits an initial reduction and largely increases after about $\varepsilon_{z}=2 \%$ for RVE A. For RVE B, $M$ stays at a constant value. The effective permeability $k$ also evolves with the porosity according to the Kozeny-Carmen relation.

Another advantage of the multiscale scheme is the accessibility to evolution of micro-structures during deformations. To demonstrate this, we perform a simple microstructural analysis in which the Anisotropic Critical State Theory (ACST) introduced by Li and Dafalias [2012], Zhao and Guo [2013], Li and Dafalias [2015] is adopted to analyze the fabric of the fluid-saturated granular assemblies at the finite strain range. The fabric anisotropy of two RVEs, one taken inside the shear band (RVE A) and another one in the host matrix (RVE B) are analyzed and compared against each other. The fabric tensor $G_{\text {fabric }}$ is contact-normalbased and is computed from a DEM RVE via Li and Dafalias [2015]

$$
G_{\text {fabric } i j}=\frac{1}{N_{c}} \sum_{c \in N_{c}} n_{i}^{c} n_{j}^{c}
$$

where $n^{c}$ is the unit vector of contact normal and $N_{c}$ is the number of contacts inside the RVE. The tensor $\boldsymbol{F}_{\text {fabric }}$ characterizes the fabric anisotropy of the RVE and is written as [Zhao and Guo, 2013]

$$
F_{\text {fabric } i j}=\frac{15}{2}\left(G_{\text {fabric } i j}-\frac{1}{3} \delta_{i j}\right)
$$

where $\delta_{i j}$ is the Kronecker delta. Its norm $F_{\text {fabric }}$ and direction $\boldsymbol{n}_{F}$ are defined by

$$
\boldsymbol{F}_{\text {fabric }}=F_{\text {fabric }} \boldsymbol{n}_{F}, \quad F_{\text {fabric }}=\sqrt{\boldsymbol{F}_{\text {fabric }}: \boldsymbol{F}_{\text {fabric }}}
$$

To analyze whether and how fabric evolves differently inside shear band and the host matrix, we compute the normalized fabric anisotropy variable (FAV) $A=\boldsymbol{n}_{F}: \boldsymbol{n}_{S}$ (a measure introduced in Li and Dafalias [2012], Zhao and Guo [2013] that quantifies the relative orientation of the tensor $\boldsymbol{F}_{\text {fabric }}$ and the deviatoric stress tensor s) for RVE A (inside shear band) and RVE B (outside shear band). The evolution of deviatoric 


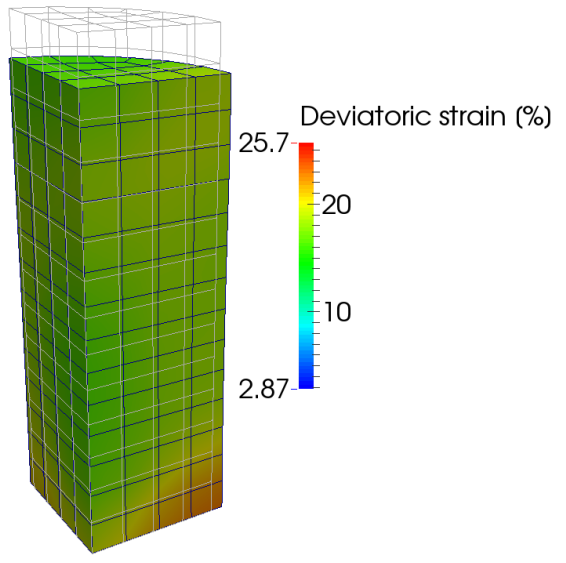

(a)

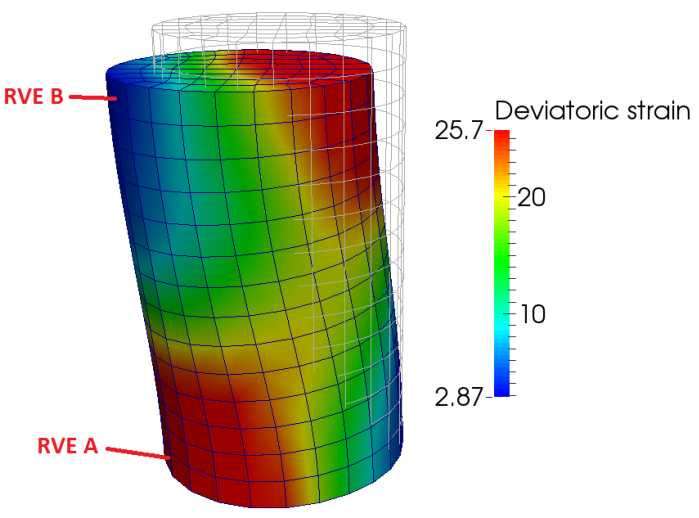

(c)

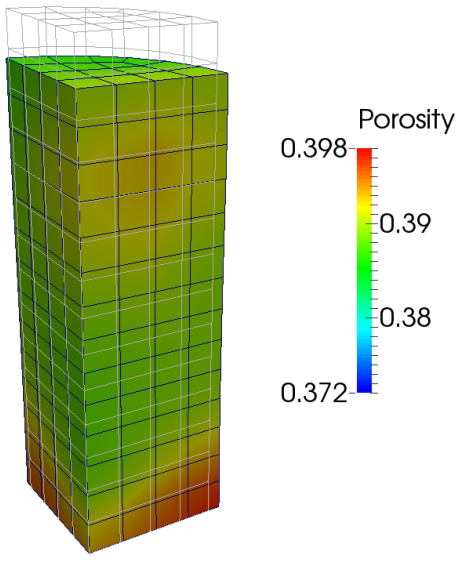

(b)

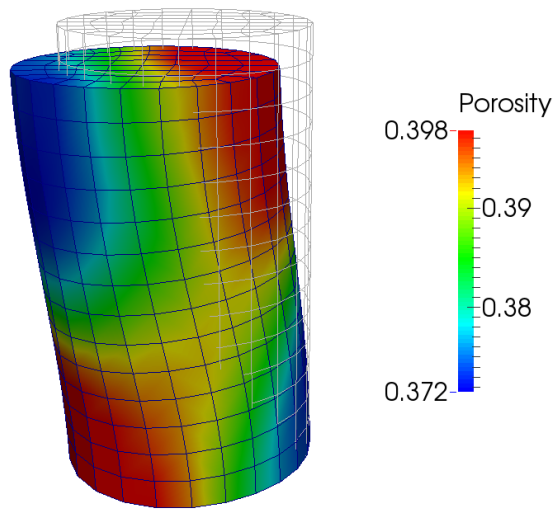

(d)

Fig. 15: Distribution of deviatoric strain and porosity in globally drained triaxial compression test at 9\% axial strain. Comparison of quarter-domain and full-domain simulations.

stress $q$ and porosity against axial strain $\varepsilon_{z}$ are also monitored to measure how close the materials in the two RVEs reach the critical state according to the anisotropic critical state theory, i.e.,

$$
\eta=\eta_{c}, e=e_{c}=\hat{e}_{c}(p) \text { and } A=A_{c}=1
$$

where $\eta$ is the ratio between the effective mean pressure $p^{\prime}$ and the deviatoric stress $q$ and $e$ is the void ratio. $\eta_{c}, e_{c}=\hat{e}_{c}(p)$ and $A_{c}=1$ are critical state values of the stress ratio, void ratio and fabric anisotropy variable (cf. Li and Dafalias [2012, 2015]).

The results are summarized in Fig. 19. The stress-strain response shown in Fig. 19(a) indicates that RVE A becomes unstable after the peak shear stress and experiences significant dilation until the critical state indicated by the plateau in the porosity curve. The normalized FAV of RVE A rises to about 0.96 quickly upon subjected to the triaxial loading. Then, normalized FAV stay close to 1, which indicates that the fabric and stress directions in RVE A is nearly coaxial, as the RVE A approaches the critical state.

On the other hand, RVE B, which lies outside the shear band, experiences slightly more softening, but the dilatancy is much less than RVE A. The FAV curve of RVE B deviates from the curve of RVE A after axial strain of $2 \%$ and exhibits opposite trend that the fabric and stress directions loss coaxiality. This 


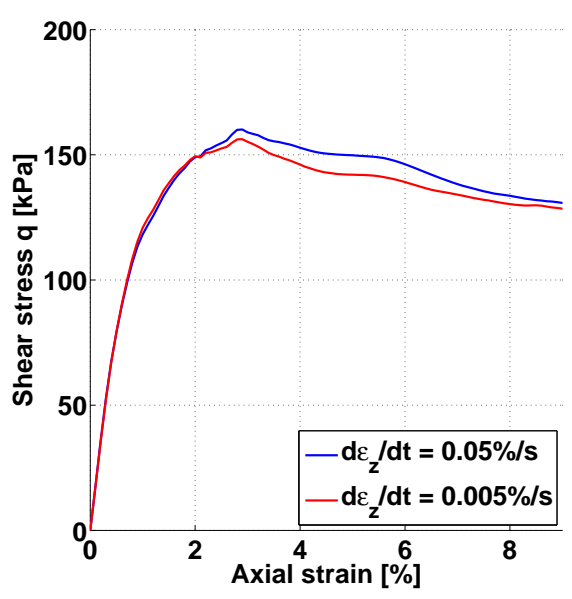

(a)

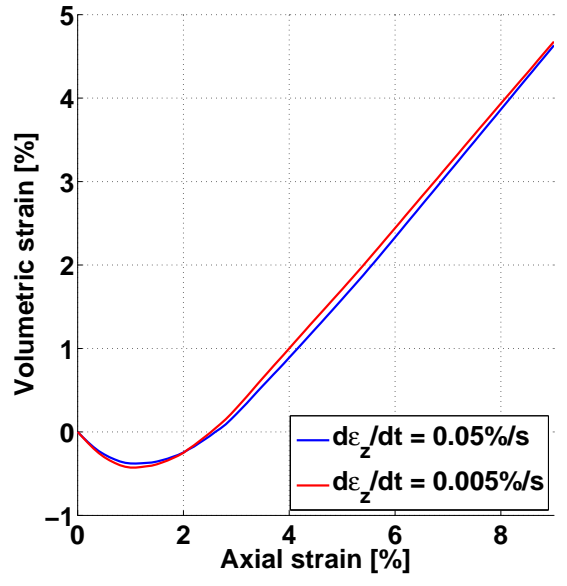

(b)

Fig. 16: Global shear stress and volumetric strain behavior in globally drained triaxial compression test. Comparison of two loading rate.

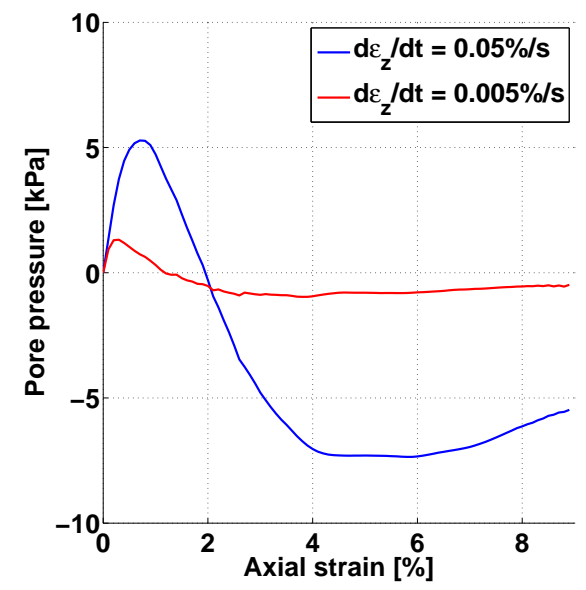

Fig. 17: Evolution of pore pressure at the center of the cylindrical specimen during triaxial compression test subjected to two loading rate.

observation suggests that the critical states are not achieved simultaneously within an specimen that forms deformation band.

To demonstrate the performance of the multiscale semi-implicit scheme, the convergence rate of the quarter-domain simulation is illustrated in Fig. 20 as an example. At different strain levels, the convergence curves show linear profiles in the logarithm-scale plot. The first step converges the fastest since the RVEs are linear elastic at $\varepsilon_{z}=0.1 \%$. The number of iterations required for convergence increases to 11 when the global shear stress reaches the peak (about $\varepsilon_{z}=2 \%$ ). In the softening stage, the explicitly treated the elastic-plastic contribution $K^{e p}$ to the material tangential stiffness becomes more significant. Therefore the convergence rate is further reduced and each time step requires about 20 iterations. 


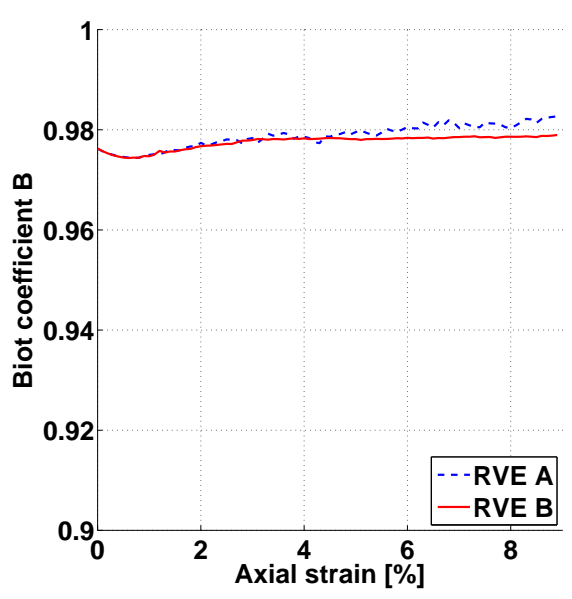

(a)

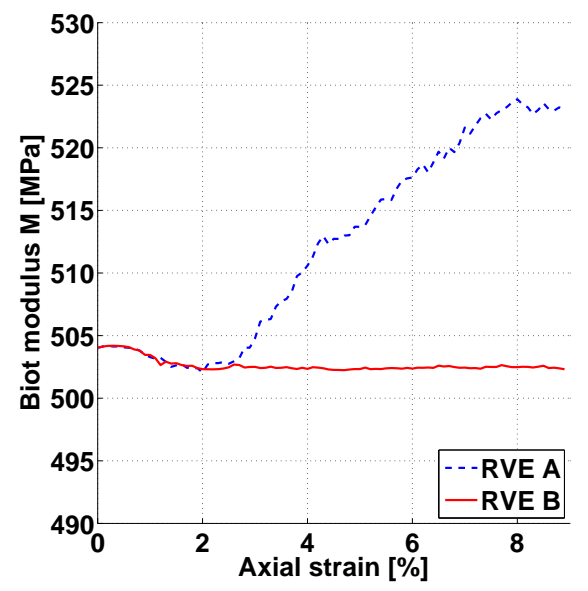

(b)

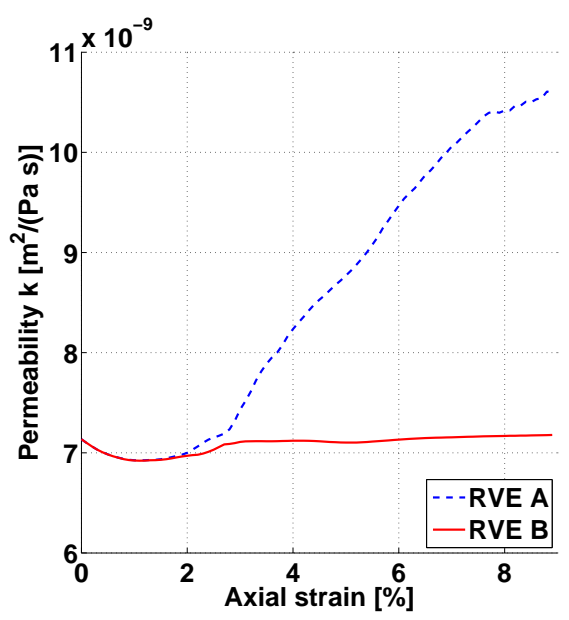

(c)

Fig. 18: Evolution of (a) Biot's coefficient, (b) Biot's modulus and (c) effective permeability for RVE A (inside shear band, Fig. 15(c)) and RVE B (outside shear band, Fig. 15(c)).

\subsection{Submerged slope stability problem}

The last numerical example is the submerged slope stability problem. We select this problem for two reasons. First, we want to showcase how to use the DEM-mixed-FEM model to obtain high fidelity responses at a localized domain of interest, while using conventional mixed FEM in the far-field domain. Second, we want to demonstrate the applicability of the proposed DEM-mixed-FEM model with a very common and simple field-scale problem.

The slope problem we consider consists of a 1:1 slope sitting on a bedrock that is assumed to be both impermeable and rigid. The slope is fully submerged underneath 2 meters of water. We conduct a classical slope stability analysis using the commercial software SLOPE/W and compare it with the results obtained from the DEM-mixed-FEM simulation. In particular, we compute the factor of safety using both the classical Bishop's method available in SLOPE/W and the proposed DEM-mixed-FEM model. The factor of safety (FOS) is defined as the ratio between the shear stress at failure $\tau_{f}$ and the current shear stress on the 


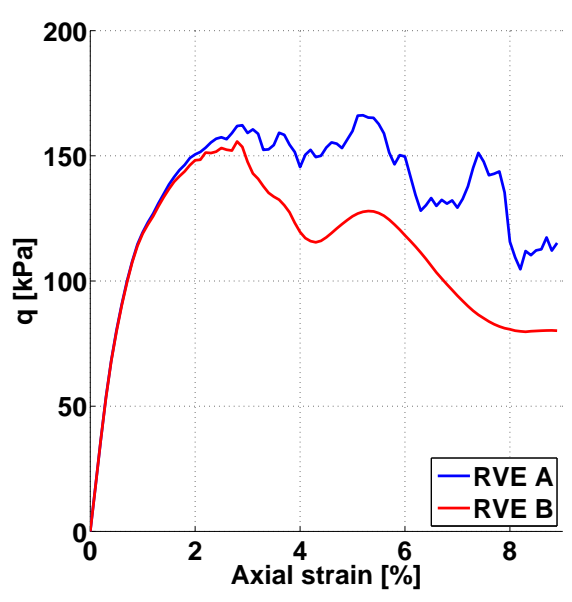

(a)

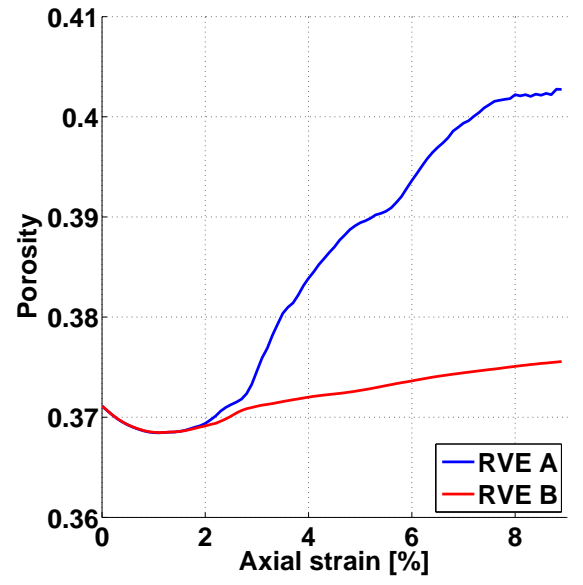

(b)

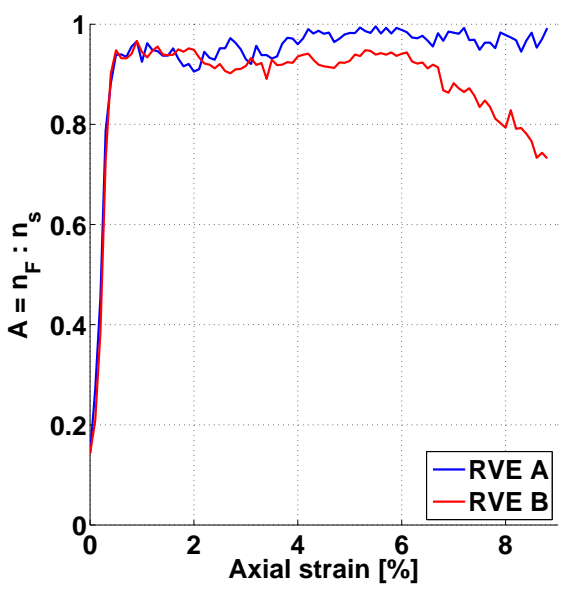

(c)

Fig. 19: Evolution of (a) deviatoric stress $q$ (b) porosity (c) $A=\boldsymbol{n}_{F}: \boldsymbol{n}_{S}$ (relative orientation between anisotropic fabric and deviatoric stress directions) during triaxial compression test $\left(\dot{\epsilon}_{z}=0.05 \% / s\right)$ for RVE A (inside shear band, Fig. 15(c)) and RVE B (outside shear band, Fig. 15(c)).

slip surface $\tau$ :

$$
F O S=\frac{\tau_{f}}{\tau}
$$

where $\tau_{f}$ is determined by the material's effective cohesion $c^{\prime}$, effective friction angle $\phi^{\prime}$, total normal stress $\sigma_{n}$, and the pore pressure $u$ as [Bishop, 1955, Borja et al., 2012]:

$$
\tau_{f}=c^{\prime}+\left(\sigma_{n}-u\right) \tan \phi^{\prime}
$$

To make the comparison between the SLOPE/W and the DEM-mixed-FEM model feasible, the DEM unit cell adopted in this simulation is firstly subjected to drained triaxial compression test under different confining pressures to obtain the Mohr-Coulomb failure envelope. The grain contact model we employed in this example is from Bourrier et al. [2013], which includes the modified frictional-normal contact law that includes cohesive normal and shear force in (73) and (74). As a result, we obtain the frictional angle $\phi^{\prime}=30^{\circ}$ and cohesion $c^{\prime}=2.5 \mathrm{kPa}$ from the simulated DEM responses. 


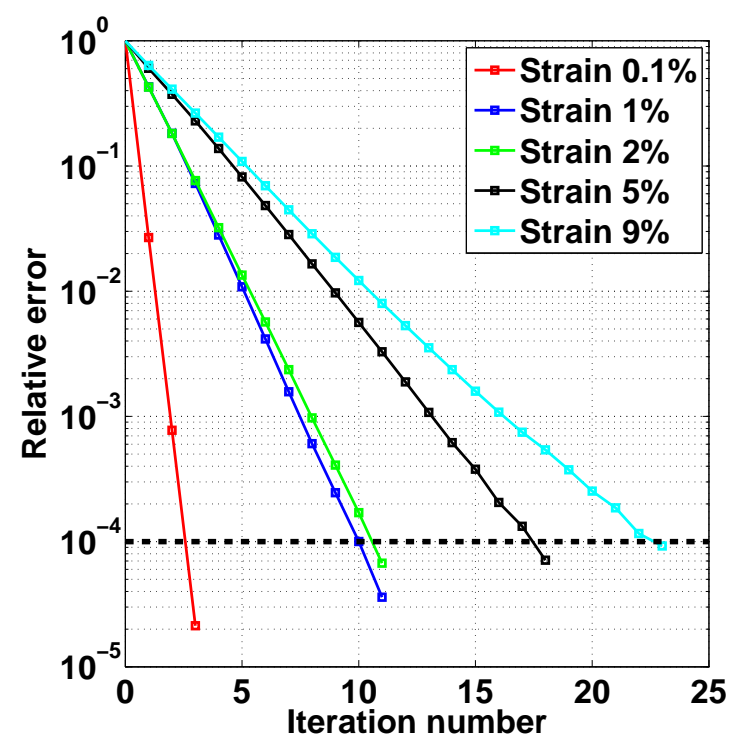

Fig. 20: Convergence profiles of the triaxial compression test at different axial strain levels. The relative error is defined as $\frac{\left\|\Delta \boldsymbol{F}^{i}\right\|}{\left\|\Delta \boldsymbol{F}^{(i=0)}\right\|}$, where $\Delta \boldsymbol{F}^{i}$ is the residual force at the iteration step $i$. The convergence is reached when the error falls below $10^{-4}$.

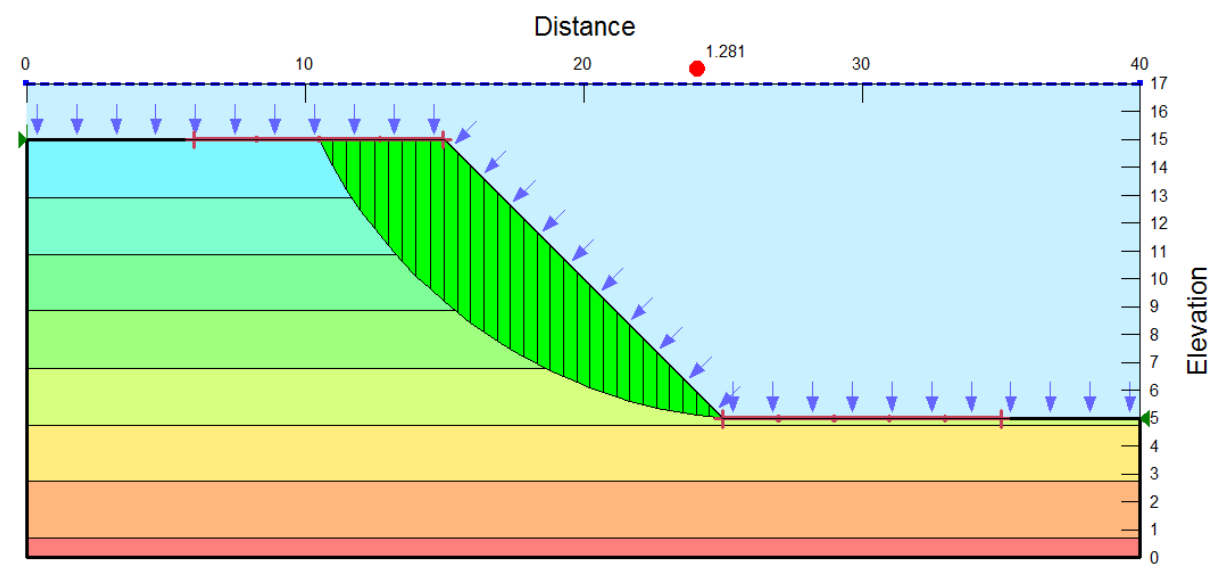

Fig. 21: Slip surface and factor of safety of 1.281 estimated by SLOPE/W for a 1:1 submerged slope. The unit of the spatial dimensions is meter.

The modified Bishop method implemented in the commercial software SLOPE/W assumes that strain localization may take place upon the slope failure and the localized zone is a circular slip surface. For a fully submerged 1:1 slope composed of materials with $\phi^{\prime}=30^{\circ}$ and $c^{\prime}=2.5 \mathrm{kPa}$, we calculate the center and radius of slip surface, as well as FOS which is assumed to be constant along the surface. The FOS computed by SLOPE/W is 1.281, as shown in Fig. 21.

The FEM-mixed-DEM simulation we conduct here assumes that the model fails with the same slip surface as the geometry calculated above. Thus only the material constitutive relation in elements along the slip surface comes from the DEM solver while as the other elements outside the critical surface are assumed to have a linear elastic behavior, possessing the same elastic properties as the DEM samples. 
Here we follow the simple approach used in Cappa and Rutqvist [2011] to model both the bulk and the critical surface with 8-node brick elements, but the the thickness of the critical surface elements are set be of the order of the material length scale (i.e. $5 \mathrm{~cm}$ ). In the future, we will explore the usage of finite strain localization element [Yang et al., 2005] or embedded localization zone model [Fish and Belytschko, 1988] to couple DEM with FEM for problems with weak or strong discontinuities. These treatments, nevertheless, are out of the scope of this study.

In this multiscale study, the factor of safety is estimated by gradually applying the gravity load $\alpha g$ at a very low rate to maintain the drained condition, where $\alpha$ is known as the loading factor [de Borst et al., 2012]. The FOS is equal to $\alpha$ when the slope suddenly collapses along the slip surface, indicated by a sudden increase of displacement of upper crest (settlement). Since a DEM assembly with neither confining pressure nor cohesive force is not stable when sheared, we start the simulation from $0.1 \mathrm{~g}$. Then $\alpha$ is increased gradually until it approaches the critical value that causes the slip surface slides. Here we chose a small increment of $\alpha(0.001 \mathrm{~g})$ in order to capture the slope response near and after the sliding. The u-p poromechanics formulation is advantageous in the sense that one may prescribe directly the pore pressure as boundary condition to represent the hydraulic load changes of groundwater level. As a result, there is no need to estimate the total traction caused by hydraulic force at the upper boundaries as what typically did in single-phase finite element analysis, e.g., Griffiths and Lane [1999].

Fig. 22 shows the distribution of accumulated plastic strain $\bar{\varepsilon}^{p}$ inside the slip surface on the verge of collapse, which is defined as:

$$
\bar{\varepsilon}^{p}=\int_{0}^{t} \sqrt{\frac{2}{3} \dot{\varepsilon}^{p}: \dot{\varepsilon}^{p}} d t=\int_{0}^{t} \sqrt{\frac{2}{3}}\left\|\dot{\varepsilon}^{p}\right\| d t=\int_{0}^{t} \sqrt{\frac{2}{3}}\left\|\dot{\varepsilon}-\dot{\varepsilon}^{e}\right\| d t=\int_{0}^{t} \sqrt{\frac{2}{3}}\left\|\dot{\varepsilon}-K^{e-1} \dot{\sigma}\right\| d t
$$

The deformed configurations of three RVEs on the top, in the middle and at the toe along the slip surface are colored according to the displacement with respect to the initial RVE configuration. Their statepaths are plotted in Fig. 23, which explain that different locations of the slip surface experience different loading pattern, the multiscale study of safety factor is thus more realistic. The safety factor predicted by the numerical analysis is shown in Fig. 24, compared with FOS predicted by the former analytical solution. The result suggests that the multiscale study yields more conservative prediction.

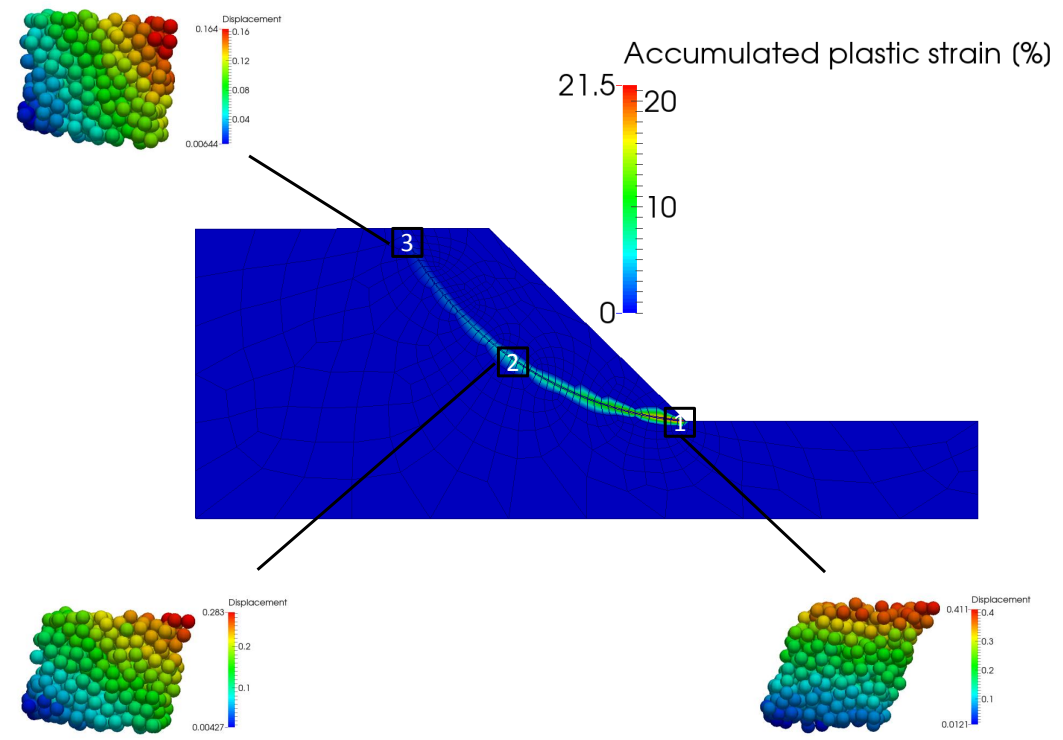

Fig. 22: Accumulated plastic strain $\bar{\varepsilon}^{p}$ inside the slip surface on the verge of collapse (Load factor $\alpha=1.23$ ) 


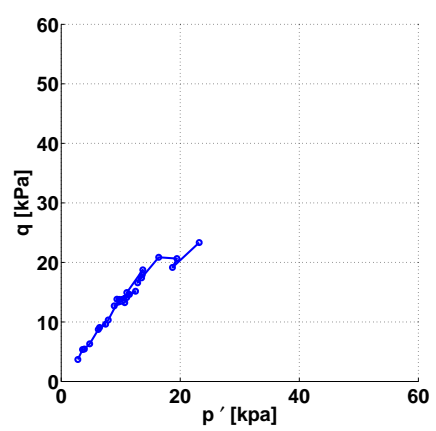

(a) RVE No.1

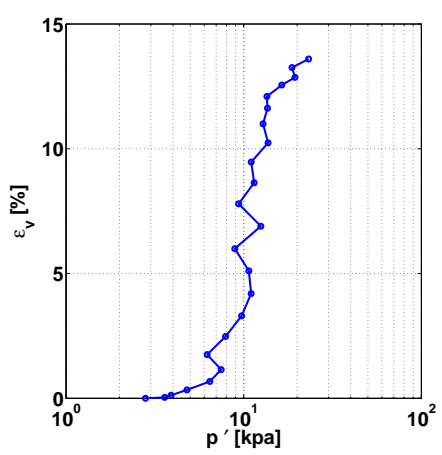

(d) RVE No.1

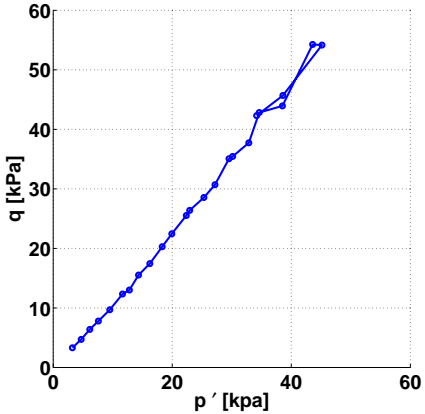

(b) RVE No.2

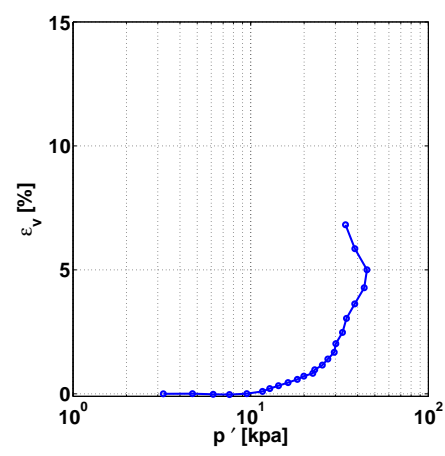

(e) RVE No.2

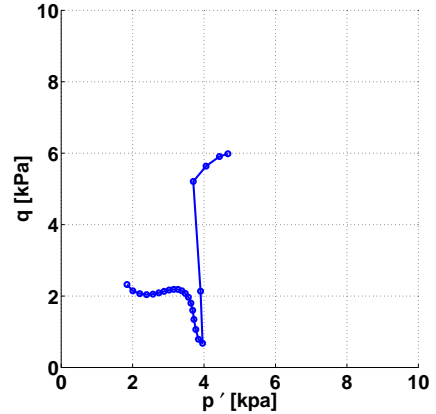

(c) RVE No.3

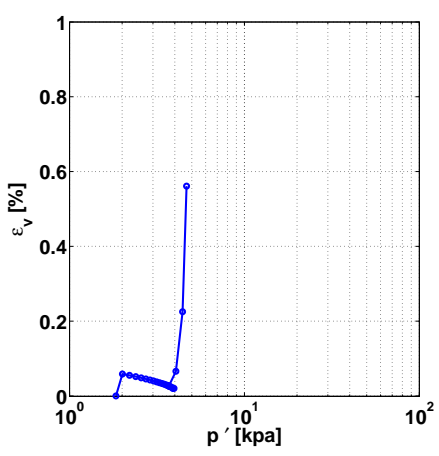

(f) RVE No.3

Fig. 23: State paths of unit cells as labeled in Fig.22 until slope collapses. Left: RVE No. 1; Middle: RVE No. 2; Right: RVE No. 3;

\section{Conclusions}

In this work, we present a finite strain dual-scale hydro-mechanical model that couples grain-scale granular simulations with a macroscopic poro-plasticity model at low Reynolds number. Using effective stress principle, the macroscopic total stress is partitioned into effective stress, which is homogenized from grainscale simulations and macroscopic pore pressure, which is updated from macroscopic simulation. To improve computational efficiency, we adopt a semi-implicit predictor-multicorrector scheme that splits the internal force into macroscopic and microscopic components. By updating the macroscopic poro-elastic contribution (FEM) implicitly and the microscopic counterpart (DEM) explicitly, we establish a multiscale scheme that is unconditionally stable and therefore allows simulations to advance in time steps large enough for practical applications. The dual-scale poromechanics model is first tested against Terzaghi's one-dimensional consolidation problem in both geometrically linear and nonlinear regimes. Additional multiscale simulations at specimen- and field-scale are also conducted to showcase the potentials of the proposed method to solve a wide spectrum of problems across spatial length scales. To the best of our knowledge, this is the first time a hierarchical multiscale coupling scheme is established to resolve finite strain poro-plasticity problem. Due to the introduction of semi-implicit scheme across length scales, the proposed method is not only suitable for specimen-level simulations, but also shown to be efficient enough to resolve field-scale problem within limited computational resource. 


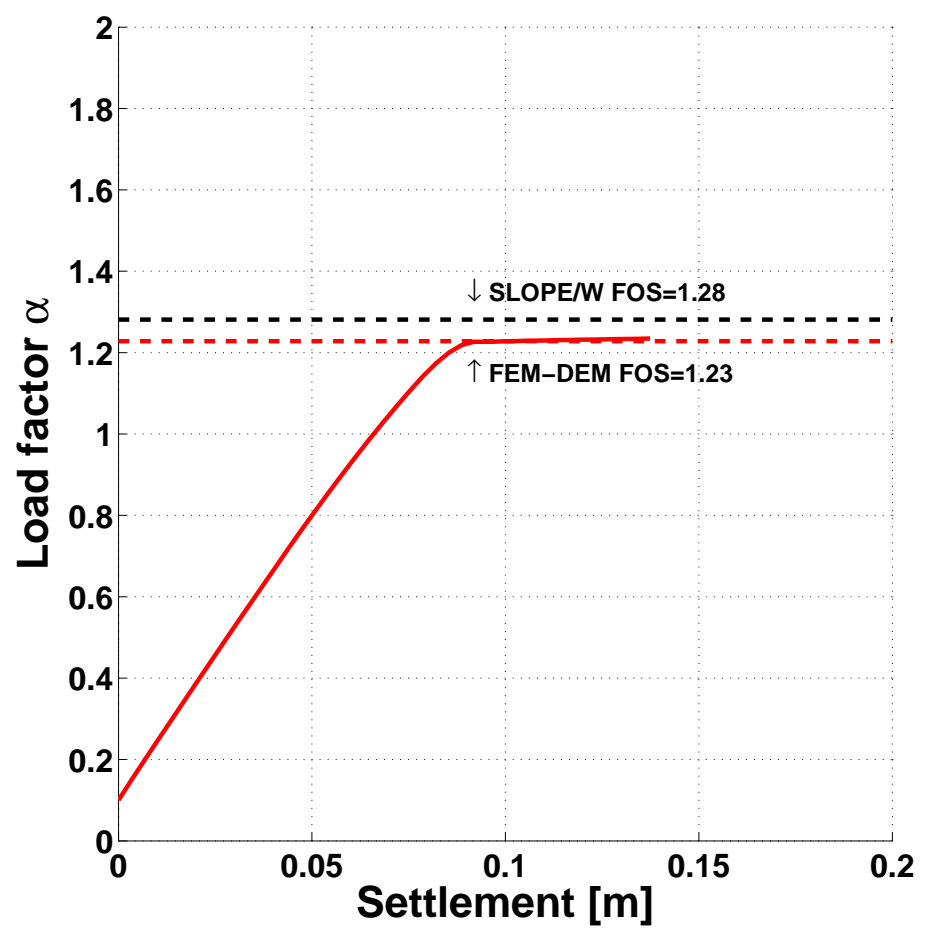

(a)

Fig. 24: Slope stability. Load-settlement diagram

\section{Acknowledgments}

This research is supported by the Earth Materials and Processes program at the US Army Research Office under grant contract W911NF-14-1-0658 and W911NF-15-1-0581, the Mechanics of Material and Structures program at National Science Foundation under grant contract CMMI-1462760 and Provost's Grants Program for Junior Faculty Diversity Grant at Columbia University. These supports are gratefully acknowledged. We are also grateful for the three anonymous reviewers for providing their insightful feedback and comments.

\section{Appendix A: Discrete element simulations at RVE}

To obtain effective stress measure from the DEM, we constitute a microscopic problem in which the macroscopic deformation measure is recast as the boundary condition for the unit problem. The unit cell problem is used to replace the macroscopic constitutive model that relates macroscopic strain measure and internal variables with the macroscopic effective stress measure. In the DEM model we employed, there is no microscopic internal variable introduced for the contact laws. Instead, path dependent behavior is mainly caused by the rearrangement of the grain contacts and the evolution of the force chain network topology.

In the unit cell DEM problem, the frame or walls of the particle assemblies are driven to move according to the macroscopic deformation measure via applying boundary traction or prescribing displacements on boundary particles [Miehe et al., 2010, Guo and Zhao, 2014]. The contact forces are computed for each particle and the equations of motion are integrated by an explicit time integrator [Cundall and Strack, 1979]. In quasi-static problems, to achieve static equilibrium of the particle assemblies, a dynamic relaxation scheme is employed. 
Consider two rigid spheres $p$ and $q$ with radii $R_{p}$ and $R_{q}$ modeling a particle pair in contact inside a granular assembly. Let $\boldsymbol{y}_{p}$ and $\boldsymbol{y}_{q}$ denote the position vectors of their centers in a global coordinate system, while their orientations are represented by unit orientation quaternions $\boldsymbol{q}_{p}$ and $\boldsymbol{q}_{q}$ [Šmilauer et al., 2010]. The relative velocity $\dot{\boldsymbol{d}}_{t}$ of the contact point $\boldsymbol{y}_{c}$ depends on the rate of change of the position vectors $\dot{y}_{p}$ and $\dot{y}_{q}$ and the rate of change of the particle orientations $w_{p}$ and $w_{q}$, i.e.,

$$
\dot{\boldsymbol{d}}_{t}=\dot{\boldsymbol{y}}_{q}-\dot{y}_{p}-\boldsymbol{w}_{q} \times\left(\boldsymbol{y}_{c}-\boldsymbol{y}_{q}\right)-\boldsymbol{w}_{p} \times\left(\boldsymbol{y}_{c}-\boldsymbol{y}_{p}\right)
$$

Assuming that the contact areas of all particle pairs are infinitesimal and neglecting the gravitational force, and we also don't consider torques/couples at contacts due to rolling and torsion in the numerical examples in this paper, the equations of motion for the translational and rotational degrees of freedom of particle $p$ reads,

$$
\left\{\begin{array}{l}
m_{p} \ddot{\boldsymbol{y}}_{p}=\boldsymbol{f}_{p}=\sum_{c}^{n_{c}} f_{p}^{c} \\
I_{p} \dot{\boldsymbol{w}}_{p}=\boldsymbol{t}_{p}=\sum_{c}^{n_{c}}\left(\boldsymbol{y}_{c}-\boldsymbol{y}_{p}\right) \times \boldsymbol{f}_{p}^{c}
\end{array}\right.
$$

with the mass $m_{p}$ and moment of inertia $I_{p}$ of the sphere $p, f_{p}$ the sum of $n_{c}$ contact forces $f_{p}^{c}$ and $\boldsymbol{t}_{p}$ the sum of $n_{c}$ contact torques due to tangential forces.

In YADE, following the form of Cundall's global damping [Cundall and Strack, 1979], artificial numerical damping forces and torques are applied on each particle to reduce the total force and total torque that increases kinematic energy, while introducing damping to all eigen-frequencies [Šmilauer et al., 2010]. The damping force $f_{p}^{\text {damp }}$ on particle $p$ is a function of the total contact force $f_{p}$, the particle velocity $\dot{\boldsymbol{y}}_{p}$ and a dimensionless damping coefficient $\lambda_{\text {damp }}$ (which we set to be 0.2 for all numerical examples presented in this paper). The damping torque $t_{p}^{\text {damp }}$ on the rotational degree of freedom is constructed in a similar way, i.e.,

$$
\left\{\begin{array}{l}
f_{p}^{\text {damp }}=-\lambda_{\text {damp }} f_{p} \operatorname{sgn}\left(f_{p} \cdot \dot{\boldsymbol{y}}_{p}\right) \\
\boldsymbol{t}_{p}^{\mathrm{damp}}=-\lambda_{\text {damp }} \boldsymbol{t}_{p} \operatorname{sgn}\left(\boldsymbol{t}_{p} \cdot \dot{\boldsymbol{w}}_{p}\right)
\end{array}\right.
$$

Finally, (65) is integrated with a central difference scheme. Consider the incremental update from time step $t$ to time step $t+\Delta t$ and let $\left(\boldsymbol{y}_{p}\right)_{t-\Delta t},\left(\boldsymbol{y}_{p}\right)_{t},\left(\boldsymbol{y}_{p}\right)_{t+\Delta t}$ denote the translational degrees of freedom for the $p$-th particle in three consecutive time steps. The explicit central difference scheme that updates $\left(y_{p}\right)_{t+\Delta t}$ reads,

$$
\left(\boldsymbol{y}_{p}\right)_{t+\Delta t}=\frac{1}{m_{p}}\left(f_{p}+f_{p}^{\mathrm{damp}}\right)_{t} \Delta t^{2}+2\left(\boldsymbol{y}_{p}\right)_{t}-\left(\boldsymbol{y}_{p}\right)_{t-\Delta t}
$$

For updating the orientation of the $p$-th particle $\left(\boldsymbol{q}_{p}\right)_{t+\Delta t}$, the explicit central difference scheme leads to the angular velocity at time $t+\frac{\Delta t}{2}$ [Šmilauer et al., 2010],

$$
\left(\boldsymbol{w}_{p}\right)_{t+\frac{\Delta t}{2}}=\left(\boldsymbol{w}_{p}\right)_{t-\frac{\Delta t}{2}}+\Delta t\left(\dot{\boldsymbol{w}}_{p}\right)_{t}=\left(\boldsymbol{w}_{p}\right)_{t-\frac{\Delta t}{2}}+\Delta t \frac{1}{I_{p}}\left(\boldsymbol{t}_{p}+\boldsymbol{t}_{p}^{\mathrm{damp}}\right)_{t}
$$

The Euler axis and angle of the rotation quaternion $\Delta \boldsymbol{q}_{p}$ are represented by a unit vector and the magnitude of the rotation $\Delta t\left(\boldsymbol{w}_{p}\right)_{t+\frac{\Delta t}{2}}$, respectively, i.e.,

$$
\left\{\begin{array}{l}
\left(\Delta \boldsymbol{q}_{p}\right)_{\boldsymbol{u}}={\widehat{\left(\boldsymbol{w}_{p}\right)_{t+\frac{\Delta t}{2}}}}_{\left(\Delta \boldsymbol{q}_{p}\right)_{\theta}=\left|\Delta t\left(\boldsymbol{w}_{p}\right)_{t+\frac{\Delta t}{2}}\right|}
\end{array}\right.
$$

The rotation quaternion $\left(\boldsymbol{q}_{p}\right)_{t+\Delta t}$ is then updated by combining two rotation quaternions together, i.e.,

$$
\left(\boldsymbol{q}_{p}\right)_{t+\Delta t}=\Delta \boldsymbol{q}_{p}\left(\boldsymbol{q}_{p}\right)_{t}
$$


Note that the multiplication of quaternions is not commutative. Following the update of particle positions and orientations, the contact forces, moment are updated and the energy balance is checked. The static equilibrium is achieved when the particle velocity becomes sufficiently small. In YADE, this is indicated by the magnitude of the kinetic energy and the unbalanced force index (cf. $\mathrm{Ng}$ [2006]).

In the actual numerical simulations, we employ a simple contact law model that can be decomposed into the normal and tangential components, $\left(f_{p}^{c}\right)^{n}$ and $\left(f_{p}^{c}\right)^{t}$ i.e.,

$$
\sum_{c}^{n_{c}} f_{p}^{c}=\sum_{c}^{n_{c}}\left(\left(f_{p}^{c}\right)^{n}+\left(f_{p}^{c}\right)^{t}\right)
$$

The normal contact force between a particle pair $p$ and $q$ is nonzero if and only if the particles are in contact, i.e.,

$$
\left(f_{p}^{c}\right)^{n}=\left(f_{p}^{c}\right)^{n} \boldsymbol{n}=\left\{\begin{array}{r}
-k_{n} d_{n} \boldsymbol{n} \text { if } d_{n} \leq 0 \\
0 \text { if } d_{n}>0
\end{array} ; d_{n}=\sqrt{\left(\boldsymbol{y}_{p}-\boldsymbol{y}_{q}\right) \cdot\left(\boldsymbol{y}_{p}-\boldsymbol{y}_{q}\right)}-R_{p}-R_{q}\right.
$$

where $n$ is the contact normal vector, $d_{n}$ is the overlapped length. Furthermore, the normal stiffness $k_{n}$ of the grain contact is related to the radii $\left(R_{p}\right.$ and $\left.R_{q}\right)$ and Young's modulus of the particle $E^{g}$, i.e.,

$$
k_{n}=2 \frac{E^{g} R_{p} R_{q}}{R_{p}+R_{q}}
$$

Meanwhile, the tangential force $\left(f_{p}^{c}\right)^{t}$ depends on the shear stiffness $k_{t}$ and the relative tangential displacement, but is also capped by the Coulomb's frictional force. As shown in Catalano et al. [2014], the rate form of the tangential constitutive law reads,

$$
\overline{\left(f_{p}^{c}\right)^{t}}=\left\{\begin{array}{r}
-k_{t} \dot{\boldsymbol{d}}_{t} \text { if } \| k_{t} \dot{\boldsymbol{d}}_{t}|| \leq\left|\left(f_{p}^{c}\right)^{n}\right| \tan (\beta) \\
0 \text { if } \| k_{t} \dot{\boldsymbol{d}}_{t}||>\left|\left(f_{p}^{c}\right)^{n}\right| \tan (\beta)
\end{array} ; k_{t}=A k_{n}\right.
$$

where $c$ is the cohesion, $A$ is a dimensionless material parameter, $\beta$ is the friction angle.

Notice that the proposed multiscale coupling model is not limited to the DEM model with this particular set of constitutive laws.

\section{Appendix B: RVE generation}

The granular assemblies used in this study is obtained using the RVE generation engine available in an open source DEM software YADE [Kozicki and Donzé, 2009]. In particular, we use the isotropic-compression method first introduced in Cundall and Strack [1979]. For completeness, we briefly outline the procedure below:

1. First, a cell box with its six faces serving as the periodic boundaries is prepared. Spheres with defined particle size distribution are then randomly inserted into the box. Initially, these inserted particles are not allowed to overlap.

2. Material parameters of particles such as the contact stiffness, density and friction angle are then assigned to the particle. At this point, the assigned inter-particle friction coefficient is set to an artificial value to manipulate the amount of particle sliding and achieve the desired porosity. A large value of friction angle will yield a loosely packed RVE, and the value is set to a very low value when a dense packing is desired.

3. The unit cell is latter subjected to isotropic compression with prescribed confining pressure. The loading is carried out by an implemented engine which is capable of controlling either the Cauchy stress or the velocity gradient of the RVE. This process terminates when the entire RVE achieves static equilibrium.

4. Finally, the real values of friction coefficient are re-assigned to all particles and the RVE is now ready for future simulations.

If frictionless rigid walls are used as the RVE driving boundary, they are simply generated in the first step to replace the periodic box. As pointed out by Jiang et al. [2003], the isotropic compression method is very efficient in generating dense granular assemblies. However, it is hard to maintain uniformity for loose specimens. 


\section{Appendix C: Successive sample reduction test for boundary condition sensitivity}

The size of the unit cell (and therefore number of particles in the RVE) determines whether the apparent responses homogenized from microstructures of RVE could give converged coarse-scale effective properties. The size of the unit cell must be sufficiently large such that the apparent responses are insensitive to the imposed boundary condition [Wellmann et al., 2008] and that it contains statistically enough mechanisms for the deformation processes. To ensure that the size of the unit cell is sufficiently large to be an RVE, we conduct a series of numerical experiments to empirically determine the representative element size. Following the ideas of successive sample tests discussed in Zohdi and Wriggers [2001], White et al. [2006], Sun et al. [2011a], we first create a large assembly composed of 979 equal-size spherical particles and obtain the local equilibrium state under a $100 \mathrm{kPa}$ isotropic compression. Then we successively reduce the size of the cubic sampling window and obtain four other granular assembles with 619, 413, 311 and 75 particles. Four of these assemblies are shown in in Fig. 25. Each RVE is then brought to equilibrium state under confining pressure of $100 \mathrm{kPa}$. To analyze whether the granular assembly is homogeneous, we also plot the Rose diagram of the contact normal orientation and show them in Fig. 25. We observed that the the contact normals are distributed quite evenly in all assemblies, except the smallest one with 75 particles. This result is consistent with the finding on 2D granular assemblies reported in [Guo and Zhao, 2014] in which a granular assembly consisting of too few particles tends to exhibit more anisotropic responses. Nevertheless, the contact normal distribution also indicates that a few hundreds of particles may be enough to generate a dense assembly with a statistically homogeneous fabric.

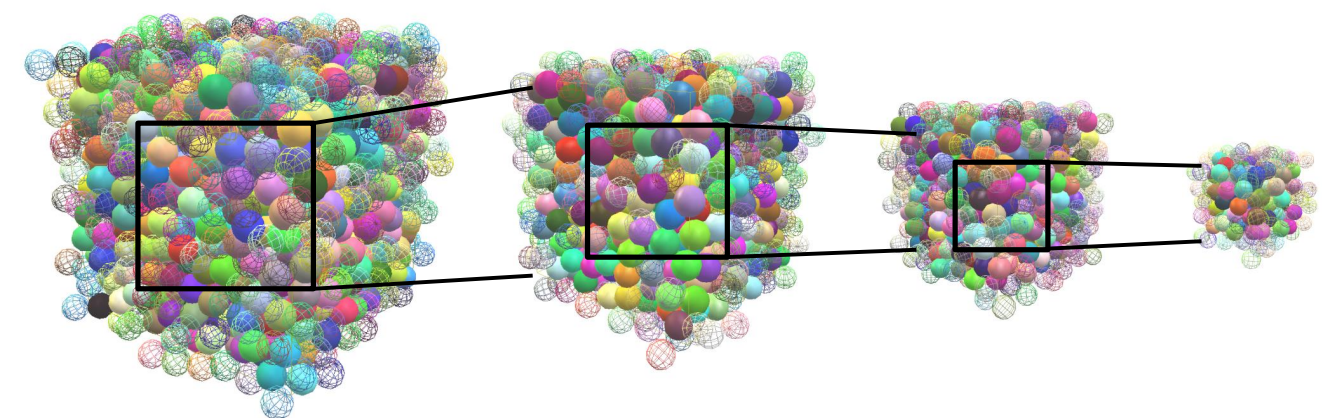

No. particles: 979

No. particles: 619

No. particles: 413

No. particles: 75
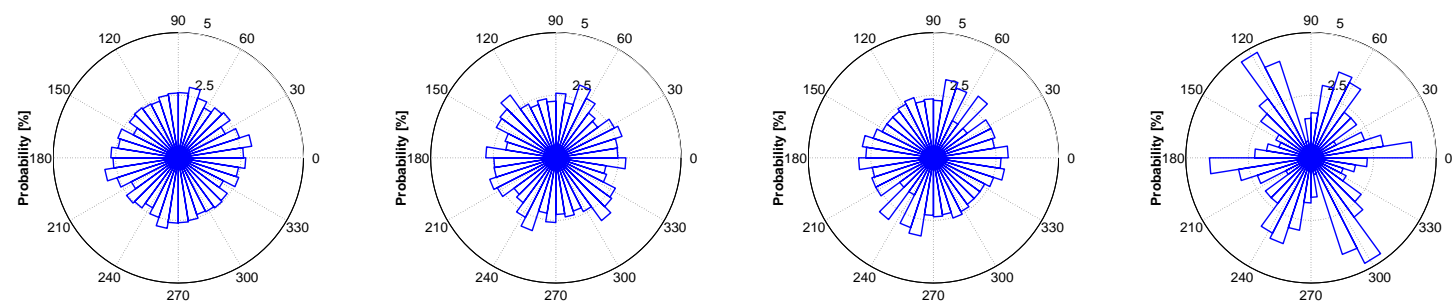

Fig. 25: Four different sizes of granular assemblies used in the RVE study (UPPER) and the corresponding Rose diagram for contact normal orientation (LOWER).

All five numerical specimens are then subjected to triaxial loading until $20 \%$ axial strain. Previous studies have established that the boundary conditions driving the frame or surrounding wall of the unit cell 
may affect the macroscopic behavior. This sensitivity to the boundary condition is more severe when the unit cell is smaller than the RVE size, but less important when the unit cell contains enough particles [Zohdi and Wriggers, 2001, Miehe and Dettmar, 2004, White et al., 2006, Wellmann et al., 2008, Miehe et al., 2010, Guo and Zhao, 2014]. In particular, Miehe et al. [2010] has conducted systematic study to compare various constraints which transform periodic, linear displacement (zero rotation) and uniform stress to particle assemblies and found that all three satisfy a priori the Hill-Mandel condition. The study in Miehe et al. [2010] demonstrates that the linear displacement constraint produces the stiffest homogenized responses, the uniform stress constraint leads to the softest homogenized responses, while the periodic constraint leads to the intermediate response which is considered the optimal choice in [Miehe and Dettmar, 2004]. In this study, we conduct numerical experiments for two types of boundary conditions, i.e. periodic boundary and frictionless rigid walls that impose linear displacement and zero rotation. The shear stress responses and porosity paths of triaxial compression tests on different size of RVEs are shown in Fig. 26.

\section{Appendix D: Matrix form of finite strain multiscale u-p formulation}

For convenience of implementation, we reformulate the finite strain multiscale u-p formulation by adopting the Voigt notations and matrices presented in [de Borst et al., 2012].

The vector for the second PK stress tensor $S$ is expressed as

$$
\{\boldsymbol{S}\}=\left[\begin{array}{llllll}
S_{11} & S_{22} & S_{33} & S_{12} & S_{23} & S_{13}
\end{array}\right]^{\mathrm{T}}
$$

and the Green-Lagrange strain tensor as

$$
\{\boldsymbol{E}\}=\left[\begin{array}{llllll}
E_{11} & E_{22} & E_{33} & 2 E_{12} & 2 E_{23} & 2 E_{13}
\end{array}\right]^{\mathrm{T}}
$$

The internal virtual work is thus expressed as:

$$
\delta W_{\text {int }}=\int_{\mathcal{B}} \delta \boldsymbol{E}: S \mathrm{dV}=\int_{\mathcal{B}}\{\delta \boldsymbol{E}\}^{\mathrm{T}}\{\boldsymbol{S}\} \mathrm{dV}
$$

The nodal displacement $\bar{u}$ and nodal pore pressure $\overline{p^{f}}$ are organized in the form

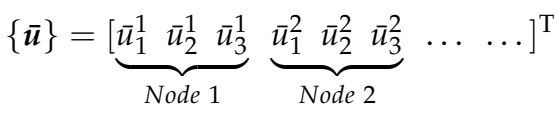

$$
\begin{aligned}
& \left\{\overline{\boldsymbol{p}}^{f}\right\}=\left[\begin{array}{llll}
\bar{p}^{f}{ }_{1} & \bar{p}^{f} & \ldots & \ldots
\end{array}\right]^{\mathrm{T}}
\end{aligned}
$$

The corresponding shape functions for displacement $N_{u}$ and pore pressure $N_{p}$ are expressed in their matrix forms as

$$
\begin{aligned}
\boldsymbol{N}_{u} & =\left[\begin{array}{cccccccc}
N_{u}^{1} & 0 & 0 & N_{u}^{2} & 0 & 0 & \ldots & \ldots \\
0 & N_{u}^{1} & 0 & 0 & N_{u}^{2} & 0 & \ldots & \ldots \\
0 & 0 & N_{u}^{1} & 0 & 0 & N_{u}^{2} & \ldots & \ldots
\end{array}\right] \\
\boldsymbol{N}_{\boldsymbol{p}} & =\left[\begin{array}{lllll}
N_{p}^{1} & N_{p}^{2} & \ldots & \ldots
\end{array}\right]
\end{aligned}
$$

The gradient of $N_{p}$ with respect to the reference configuration reads:

$$
\boldsymbol{E}_{\boldsymbol{p}}=\nabla^{X} \boldsymbol{N}_{\boldsymbol{p}}=\left[\begin{array}{lllll}
\frac{\partial N_{p}^{1}}{\partial X_{1}} & \frac{\partial N_{p}^{2}}{\partial X_{1}} & \ldots & \ldots \\
\frac{\partial N_{p}^{1}}{\partial X_{2}} & \frac{\partial N_{p}^{2}}{\partial X_{2}} & \ldots & \ldots \\
\frac{\partial N_{p}^{1}}{\partial X_{3}} & \frac{\partial N_{p}^{2}}{\partial X_{3}} & \ldots & \ldots
\end{array}\right]
$$

The strain-displacement matrix $\boldsymbol{B}_{\boldsymbol{L}}$ in the total Lagrangian formulation is defined as

$$
\{\delta \boldsymbol{E}\}=\boldsymbol{B}_{\boldsymbol{L}}\{\delta \overline{\boldsymbol{u}}\}
$$




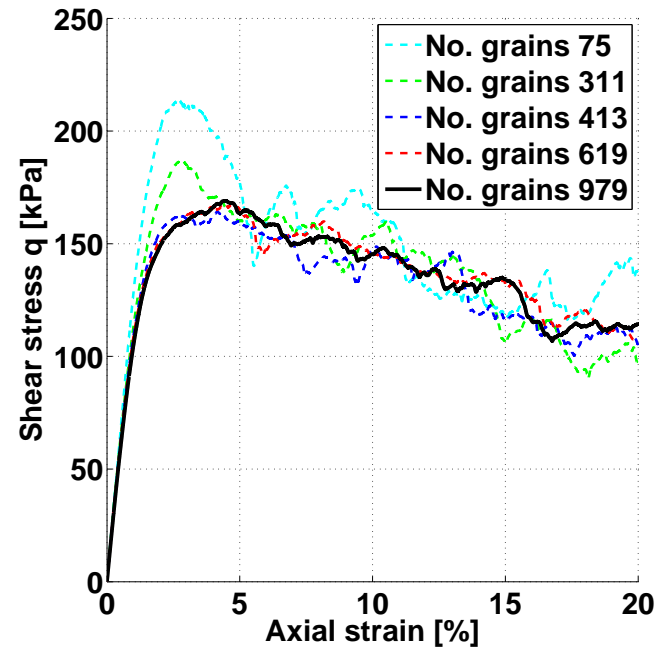

(a)

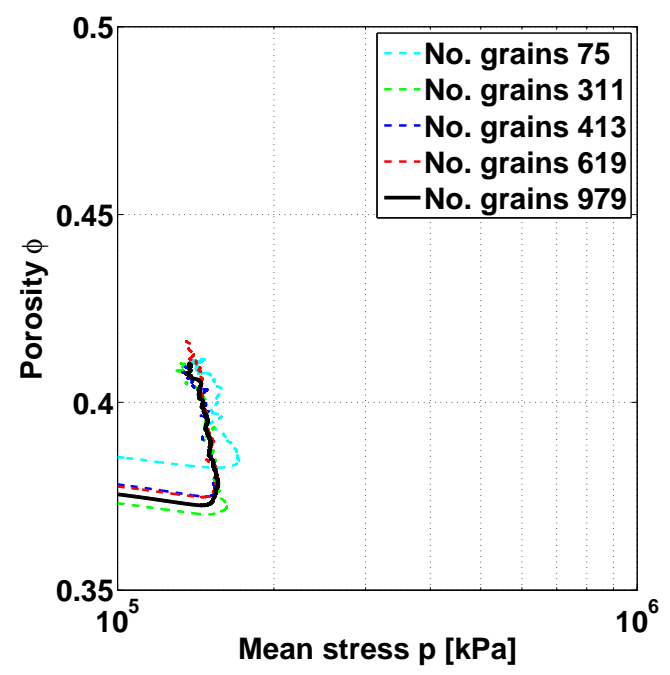

(c)

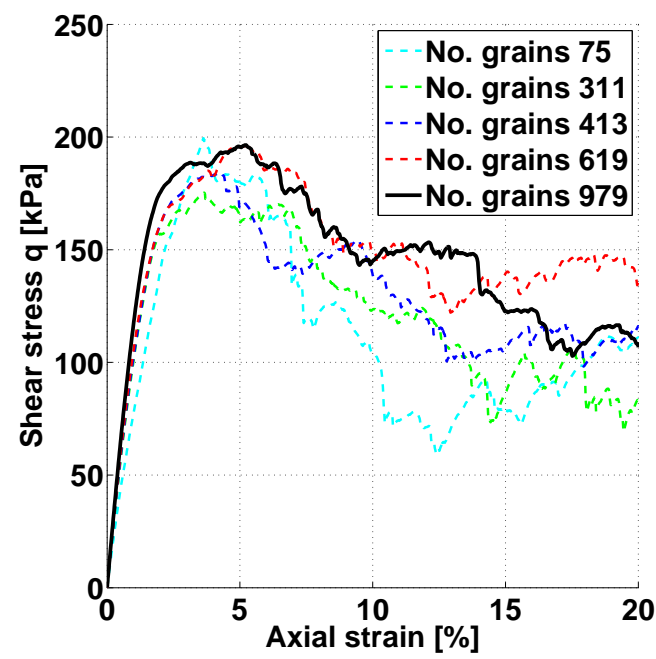

(b)

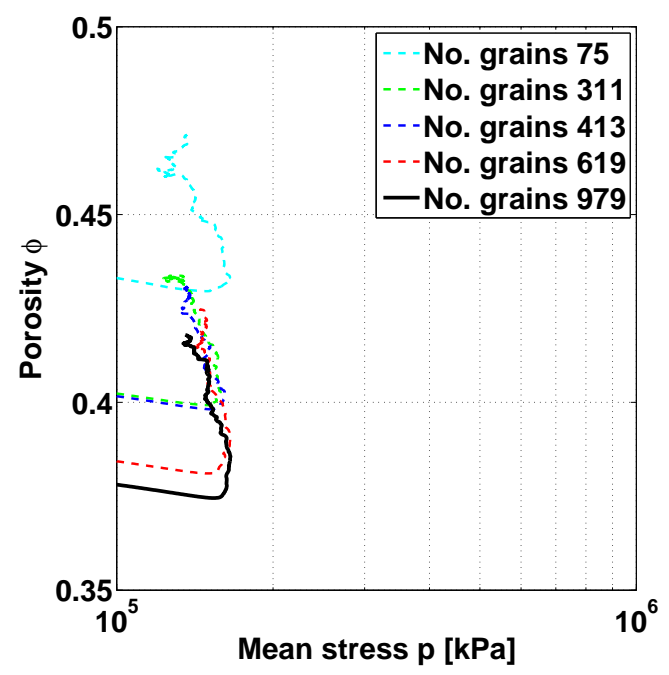

(d)

Fig. 26: Shear stress response and porosity path of triaxial compression tests on different size of RVEs. (a)(c) RVE driven by periodic boundaries; (b)(d) RVE driven by frictionless rigid walls

and its explicit expression writes

$$
\boldsymbol{B}_{L}=\left[\begin{array}{ccccc}
F_{11} \frac{\partial N_{u}^{1}}{X_{1}} & F_{21} \frac{\partial N_{u}^{1}}{X_{1}} & F_{31} \frac{\partial N_{u}^{1}}{X_{1}} & \ldots & \ldots \\
F_{12} \frac{\partial N_{u}^{1}}{X_{2}} & F_{22} \frac{\partial N_{u}^{1}}{X_{2}} & F_{32} \frac{\partial N_{u}^{1}}{X_{2}} & \ldots & \ldots \\
F_{13} \frac{\partial N_{u}^{1}}{X_{3}} & F_{23} \frac{\partial N_{u}^{1}}{X_{3}} & F_{33} \frac{\partial N_{u}^{1}}{X_{3}} & \ldots & \ldots \\
F_{11} \frac{\partial N_{u}^{1}}{X_{2}}+F_{12} \frac{\partial N_{u}^{1}}{X_{1}} & F_{21} \frac{\partial N_{u}^{1}}{X_{2}}+F_{22} \frac{\partial N_{u}^{1}}{X_{1}} & F_{31} \frac{\partial N_{u}^{1}}{X_{2}}+F_{32} \frac{\partial N_{u}^{1}}{X_{1}} & \ldots & \ldots \\
F_{12} \frac{\partial N_{u}^{1}}{X_{3}}+F_{13} \frac{\partial N_{u}^{1}}{X_{2}} & F_{22} \frac{\partial N_{u}^{1}}{X_{3}}+F_{23} \frac{\partial N_{u}^{1}}{X_{2}} & F_{32} \frac{\partial N_{u}^{1}}{X_{3}}+F_{33} \frac{\partial N_{u}^{1}}{X_{2}} & \ldots & \ldots \\
F_{13} \frac{\partial N_{u}^{1}}{X_{1}}+F_{11} \frac{\partial N_{u}^{1}}{X_{3}} & F_{23} \frac{\partial N_{u}^{1}}{X_{1}}+F_{21} \frac{\partial N_{u}^{1}}{X_{3}} & F_{33} \frac{\partial N_{u}^{1}}{X_{1}}+F_{31} \frac{\partial N_{u}^{1}}{X_{3}} & \ldots & \ldots
\end{array}\right]
$$


with the symmetric strain tensor $\boldsymbol{C}^{-1}=\left(\boldsymbol{F}^{\mathrm{T}} \boldsymbol{F}\right)^{-1}$ written in the vector form as:

$$
\left\{C^{-1}\right\}=\left[\begin{array}{llllll}
C_{11}^{-1} & C_{22}^{-1} & C_{33}^{-1} & C_{12}^{-1} & C_{23}^{-1} & C_{13}^{-1}
\end{array}\right]^{\mathrm{T}}
$$

The coupling matrix $K^{u p}$ is constructed in a similar manner and its matrix form reads

$$
\boldsymbol{K}^{u p}=\int_{\mathcal{B}} J B^{\mathrm{DEM}} \boldsymbol{B}_{L}^{\mathrm{T}}\left\{\boldsymbol{C}^{-\mathbf{1}}\right\} \boldsymbol{N}_{p} \mathrm{dV}
$$


Finally, the forces and matrices employed for implementation of the finite strain multiscale $\mathrm{u}-\mathrm{p}$ formulation are recapitulated

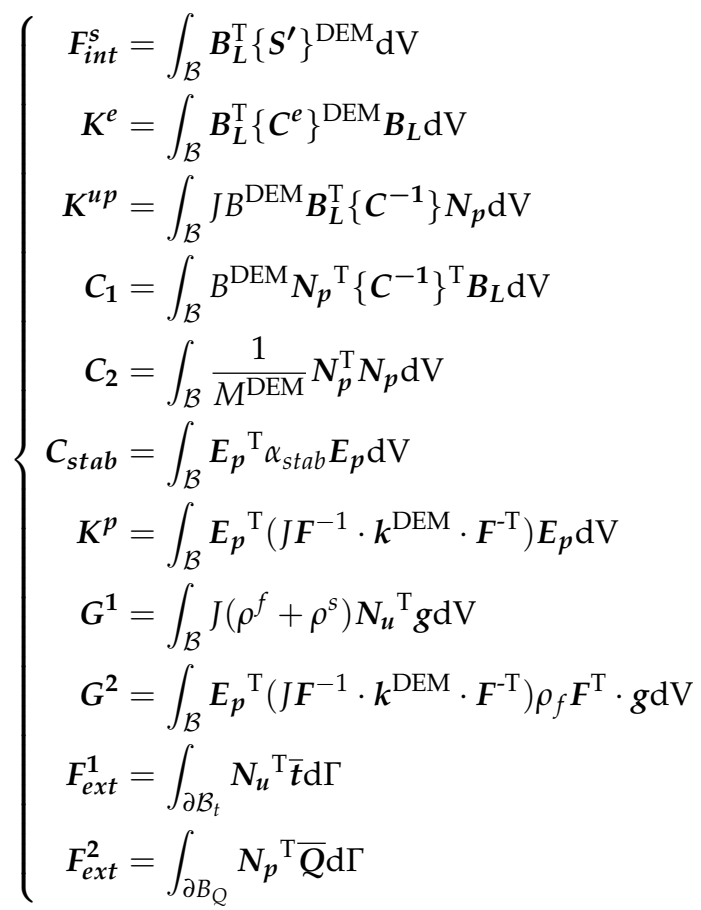

\section{References}

Francisco Armero. Formulation and finite element implementation of a multiplicative model of coupled poro-plasticity at finite strains under fully saturated conditions. Computer methods in applied mechanics and engineering, 171(3):205-241, 1999.

Katalin Bagi. Stress and strain in granular assemblies. Mechanics of materials, 22(3):165-177, 1996.

R Berger, C Kloss, A Kohlmeyer, and S Pirker. Hybrid parallelization of the liggghts open-source dem code. Powder Technology, 278:234-247, 2015.

Maurice A Biot. General theory of three-dimensional consolidation. Journal of applied physics, 12(2):155-164, 1941.

Alan W Bishop. The use of the slip circle in the stability analysis of slopes. Geotechnique, (5):7-17, 1955.

Ronaldo I Borja and Enrique Alarcón. A mathematical framework for finite strain elastoplastic consolidation part 1: Balance laws, variational formulation, and linearization. Computer Methods in Applied Mechanics and Engineering, 122(1):145-171, 1995.

Ronaldo I Borja, Claudio Tamagnini, and Enrique Alarcón. Elastoplastic consolidation at finite strain part 2: finite element implementation and numerical examples. Computer Methods in Applied Mechanics and Engineering, 159(1):103-122, 1998.

Ronaldo I Borja, Joshua A White, Xiaoyu Liu, and Wei Wu. Factor of safety in a partially saturated slope inferred from hydro-mechanical continuum modeling. International Journal for Numerical and Analytical Methods in Geomechanics, 36(2):236-248, 2012.

Franck Bourrier, François Kneib, Bruno Chareyre, and Thierry Fourcaud. Discrete modeling of granular soils reinforcement by plant roots. Ecological Engineering, 61:646-657, 2013.

Ray M Bowen. Incompressible porous media models by use of the theory of mixtures. International Journal of Engineering Science, 18(9):1129-1148, 1980.

Ray M Bowen. Compressible porous media models by use of the theory of mixtures. International Journal of Engineering Science, 20(6):697-735, 1982.

Susanne C Brenner and Ridgway Scott. The mathematical theory of finite element methods, volume 15. Springer Science \& Business Media, 2008. 
Michael D Brothers, John T Foster, and Harry R Millwater. A comparison of different methods for calculating tangent-stiffness matrices in a massively parallel computational peridynamics code. Computer Methods in Applied Mechanics and Engineering, 279:247-267, 2014.

Frédéric Cappa and Jonny Rutqvist. Modeling of coupled deformation and permeability evolution during fault reactivation induced by deep underground injection of co 2. International Journal of Greenhouse Gas Control, 5(2):336-346, 2011.

Emanuele Catalano, Bruno Chareyre, and Eric Barthélemy. Pore-scale modeling of fluid-particles interaction and emerging poromechanical effects. International Journal for Numerical and Analytical Methods in Geomechanics, 38(1):51-71, 2014.

J Christoffersen, MM Mehrabadi, and S Nemat-Nasser. A micromechanical description of granular material behavior. Journal of Applied Mechanics, 48(2):339-344, 1981.

Olivier Coussy. Poromechanics. John Wiley \& Sons, 2004.

Xilin Cui, Jun Li, Andrew Chan, and David Chapman. Coupled dem-lbm simulation of internal fluidisation induced by a leaking pipe. Powder Technology, 254:299-306, 2014.

Peter A Cundall and Otto DL Strack. A discrete numerical model for granular assemblies. Geotechnique, 29 (1):47-65, 1979.

Jennifer Sinclair Curtis and Berend Van Wachem. Modeling particle-laden flows: A research outlook. AIChE journal, 50(11):2638-2645, 2004.

Yannis F Dafalias and Majid T Manzari. Simple plasticity sand model accounting for fabric change effects. Journal of Engineering mechanics, 130(6):622-634, 2004.

René de Borst, Mike A Crisfield, Joris JC Remmers, and Clemens V Verhoosel. Nonlinear finite element analysis of solids and structures. John Wiley \& Sons, 2012.

Usama El Shamy and Mourad Zeghal. Coupled continuum-discrete model for saturated granular soils. Journal of engineering mechanics, 131(4):413-426, 2005.

J Fish and T Belytschko. Elements with embedded localization zones for large deformation problems. Computers $\mathcal{E}$ Structures, 30(1):247-256, 1988.

Jacob Fish. Practical multiscaling. John Wiley \& Sons, 2013.

SA Galindo-Torres, A Scheuermann, HB Mühlhaus, and DJ Williams. A micro-mechanical approach for the study of contact erosion. Acta Geotechnica, 10(3):357-368, 2013.

IM Gitman, H Askes, and LJ Sluys. Representative volume: existence and size determination. Engineering fracture mechanics, 74(16):2518-2534, 2007.

Christoph Goniva, Christoph Kloss, Alice Hager, and Stefan Pirker. An open source cfd-dem perspective. In Proceedings of OpenFOAM workshop Gothenburg, Sweden, 2010.

William G Gray, Bernhard A Schrefler, and Francesco Pesavento. The solid phase stress tensor in porous media mechanics and the hill-mandel condition. Journal of the Mechanics and Physics of Solids, 57(3): 539-554, 2009.

William G Gray, Cass T Miller, and Bernhard A Schrefler. Averaging theory for description of environmental problems: What have we learned? Advances in water resources, 51:123-138, 2013.

DV Griffiths and PA Lane. Slope stability analysis by finite elements. Geotechnique, 49(3):387-403, 1999.

Ning Guo and Jidong Zhao. A coupled fem/dem approach for hierarchical multiscale modelling of granular media. International Journal for Numerical Methods in Engineering, 99(11):789-818, 2014.

K Han, YT Feng, and DRJ Owen. Numerical simulations of irregular particle transport in turbulent flows using coupled lbm-dem. Computer Modeling in Engineering and Sciences, 18(2):87, 2007.

Yanhui Han and Peter A Cundall. Lattice boltzmann modeling of pore-scale fluid flow through idealized porous media. International Journal for Numerical Methods in Fluids, 67(11):1720-1734, 2011.

Yanhui Han and Peter A Cundall. Lbm-dem modeling of fluid-solid interaction in porous media. International Journal for Numerical and Analytical Methods in Geomechanics, 37(10):1391-1407, 2013.

Thomas JR Hughes, Karl S Pister, and Robert L Taylor. Implicit-explicit finite elements in nonlinear transient analysis. Computer Methods in Applied Mechanics and Engineering, 17:159-182, 1979.

TJR Hughes. The finite element method: linear static and dynamic finite element analysis. 1987.

Boris Jeremić, Zhao Cheng, Mahdi Taiebat, and Yannis Dafalias. Numerical simulation of fully saturated porous materials. International Journal for Numerical and Analytical Methods in Geomechanics, 32(13):16351660, 2008. 
MJ Jiang, JM Konrad, and S Leroueil. An efficient technique for generating homogeneous specimens for dem studies. Computers and geotechnics, 30(7):579-597, 2003.

J Kozicki and FV Donzé. Yade-open dem: an open-source software using a discrete element method to simulate granular material. Engineering Computations, 26(7):786-805, 2009.

Matthew R Kuhn, Hannah E Renken, Austin D Mixsell, and Steven L Kramer. Investigation of cyclic liquefaction with discrete element simulations. Journal of Geotechnical and Geoenvironmental Engineering, 140(12):04014075, 2014.

Chao Li, Ronaldo I Borja, and Richard A Regueiro. Dynamics of porous media at finite strain. Computer Methods in Applied Mechanics and Engineering, 193(36):3837-3870, 2004.

Xiang Song Li and Yannis F Dafalias. Anisotropic critical state theory: role of fabric. Journal of Engineering Mechanics, 138(3):263-275, 2012.

XS Li and YF Dafalias. Dissipation consistent fabric tensor definition from dem to continuum for granular media. Journal of the Mechanics and Physics of Solids, 78:141-153, 2015.

Hoe I Ling, Dongyi Yue, Victor N Kaliakin, and Nickolas J Themelis. Anisotropic elastoplastic bounding surface model for cohesive soils. Journal of engineering mechanics, 128(7):748-758, 2002.

Yang Liu, WaiChing Sun, Zifeng Yuan, and Jacob Fish. A nonlocal multiscale discrete-continuum model for predicting mechanical behavior of granular materials. International Journal for Numerical Methods in Engineering, 2015.

Mouloud Mansouri, J-Y Delenne, Moulay Saïd El Youssoufi, and Ahcene Séridi. A 3d dem-lbm approach for the assessment of the quick condition for sands. Comptes Rendus Mécanique, 337(9):675-681, 2009.

Majid T Manzari and Yannis F Dafalias. A critical state two-surface plasticity model for sands. Geotechnique, 47(2):255-272, 1997.

Mario J Martinez, Pania Newell, Joseph E Bishop, and DZ Turner. Coupled multiphase flow and geomechanics model for analysis of joint reactivation during co 2 sequestration operations. International Journal of Greenhouse Gas Control, 17:148-160, 2013.

C Miehe, J Dettmar, and D Zäh. Homogenization and two-scale simulations of granular materials for different microstructural constraints. International Journal for Numerical Methods in Engineering, 83(8-9): 1206-1236, 2010.

Christian Miehe and Joachim Dettmar. A framework for micro-macro transitions in periodic particle aggregates of granular materials. Computer Methods in Applied Mechanics and Engineering, 193(3):225-256, 2004.

Tang-Tat Ng. Input parameters of discrete element methods. Journal of Engineering Mechanics, 132(7):723$729,2006$.

Michał Nitka, Gaël Combe, Cristian Dascalu, and Jacques Desrues. Two-scale modeling of granular materials: a dem-fem approach. Granular Matter, 13(3):277-281, 2011.

Amos Nur and JD Byerlee. An exact effective stress law for elastic deformation of rock with fluids. Journal of Geophysical Research, 76(26):6414-6419, 1971.

E Onate and J Rojek. Combination of discrete element and finite element methods for dynamic analysis of geomechanics problems. Computer methods in applied mechanics and engineering, 193(27):3087-3128, 2004.

Juan M Pestana and Andrew J Whittle. Formulation of a unified constitutive model for clays and sands. International Journal for Numerical and Analytical Methods in Geomechanics, 23(12):1215-1243, 1999.

J. H. Prevost. Nonlinear transient phenomena in saturated porous media. Computer Methods in Applied Mechanics and Engineering, 30(1):3 - 18, 1982. ISSN 0045-7825.

Jean H Prevost. Implicit-explicit schemes for nonlinear consolidation. Computer Methods in Applied Mechanics and Engineering, 39(2):225-239, 1983.

RA Regueiro and D Ebrahimi. Implicit dynamic three-dimensional finite element analysis of an inelastic biphasic mixture at finite strain: part 1: application to a simple geomaterial. Computer Methods in Applied Mechanics and Engineering, 199(29):2024-2049, 2010.

James R Rice and Michael P Cleary. Some basic stress diffusion solutions for fluid-saturated elastic porous media with compressible constituents. Reviews of Geophysics, 14(2):227-241, 1976.

Martin Robinson, Marco Ramaioli, and Stefan Luding. Fluid-particle flow simulations using two-waycoupled mesoscale sph-dem and validation. International journal of multiphase flow, 59:121-134, 2014.

Lorenzo Sanavia, BA Schrefler, and P Steinmann. A formulation for an unsaturated porous medium undergoing large inelastic strains. Computational Mechanics, 28(2):137-151, 2002. 
Andrew Schofield and Peter Wroth. Critical state soil mechanics. 1968.

B.R. Simon, J.S.-S. Wu, O.C. Zienkiewicz, and D.K. Paul. Evaluation of $u-w$ and $u-\pi$ finite element methods for the dynamic response of saturated porous media using one-dimensional models. International Journal for Numerical and Analytical Methods in Geomechanics, 10(5):461-482, 1986. ISSN 1096-9853.

V Šmilauer, E Catalano, B Chareyre, S Dorofeenko, J Duriez, A Gladky, J Kozicki, C Modenese, L Scholtès, L Sibille, et al. Yade documentation. The Yade Project.(http://yade-dem. org/doc/), 2010.

WaiChing Sun. A stabilized finite element formulation for monolithic thermo-hydro-mechanical simulations at finite strain. International Journal for Numerical Methods in Engineering, 2015. ISSN 1097-0207. doi: 10.1002/nme.4910.

WaiChing Sun, Jose E Andrade, and John W Rudnicki. Multiscale method for characterization of porous microstructures and their impact on macroscopic effective permeability. International Journal for Numerical Methods in Engineering, 88(12):1260-1279, 2011a.

WaiChing Sun, José E Andrade, John W Rudnicki, and Peter Eichhubl. Connecting microstructural attributes and permeability from $3 \mathrm{~d}$ tomographic images of in situ shear-enhanced compaction bands using multiscale computations. Geophysical Research Letters, 38(10), $2011 \mathrm{~b}$.

WaiChing Sun, Matthew R Kuhn, and John W Rudnicki. A multiscale dem-lbm analysis on permeability evolutions inside a dilatant shear band. Acta Geotechnica, 8(5):465-480, 2013a.

WaiChing Sun, Jakob T Ostien, and Andrew G Salinger. A stabilized assumed deformation gradient finite element formulation for strongly coupled poromechanical simulations at finite strain. International Journal for Numerical and Analytical Methods in Geomechanics, 37(16):2755-2788, 2013b.

Karl Terzaghi, Karl Terzaghi, Civil Engineer, Austria Czechoslowakia, Karl Terzaghi, Ingénieur Civil, Autriche Tchécoslovaquie, and Etats Unis. Theoretical soil mechanics, volume 18. Wiley New York, 1943.

von K Terzaghi. The shearing resistance of saturated soils and the angle between the planes of shear. In Proceedings of the 1st International Conference on Soil Mechanics and Foundation Engineering, volume 1, pages 54-56. Harvard University Press Cambridge, MA, 1936.

Clifford Truesdell and Richard Toupin. The classical field theories. Springer, 1960.

Andrzej Truty and Thomas Zimmermann. Stabilized mixed finite element formulations for materially nonlinear partially saturated two-phase media. Computer methods in applied mechanics and engineering, 195(13):1517-1546, 2006.

C Wellmann, C Lillie, and P Wriggers. Homogenization of granular material modeled by a threedimensional discrete element method. Computers and Geotechnics, 35(3):394-405, 2008.

Joshua A White and Ronaldo I Borja. Stabilized low-order finite elements for coupled soliddeformation/fluid-diffusion and their application to fault zone transients. Computer Methods in Applied Mechanics and Engineering, 197(49):4353-4366, 2008.

Joshua A White, Ronaldo I Borja, and Joanne T Fredrich. Calculating the effective permeability of sandstone with multiscale lattice boltzmann/finite element simulations. Acta Geotechnica, 1(4):195-209, 2006.

David Muir Wood. Soil behaviour and critical state soil mechanics. Cambridge university press, 1990.

Nicolay Nicolayevich Yanenko. The method of fractional steps. Springer, 1971.

Q Yang, A Mota, and M Ortiz. A class of variational strain-localization finite elements. International Journal for Numerical Methods in Engineering, 62(8):1013-1037, 2005.

Mitsutoshi Yoshimine, Kenji Ishihara, and William Vargas. Effects of principal stress direction on intermediate principal stress on undrained shear behavior of sand. Soils and Founcations, 38(3):179-188, 1998. doi: $10.3208 /$ sandf.38.3_179.

Jidong Zhao and Ning Guo. Unique critical state characteristics in granular media considering fabric anisotropy. Géotechnique, 63(8):695-704, 2013.

HP Zhu, ZY Zhou, RY Yang, and AB Yu. Discrete particle simulation of particulate systems: theoretical developments. Chemical Engineering Science, 62(13):3378-3396, 2007.

TI Zohdi and P Wriggers. Computational micro-macro material testing. Archives of Computational Methods in Engineering, 8(2):131-228, 2001. 